\title{
Spacetime duality between localization transitions and measurement-induced transitions
}

\author{
Tsung-Cheng Lu๑* and Tarun Grover \\ Department of Physics, University of California at San Diego, La Jolla, California 92093, USA
}

(Received 10 April 2021; accepted 14 September 2021; published 28 October 2021)

\begin{abstract}
Time evolution of quantum many-body systems typically leads to a state with maximal entanglement allowed by symmetries. Two distinct routes to impede entanglement growth are inducing localization via spatial disorder, or subjecting the system to nonunitary evolution, e.g., via projective measurements. Here we employ the idea of spacetime rotation of a circuit to explore the relation between systems that fall into these two classes. In particular, by spacetime rotating unitary Floquet circuits that display a localization transition, we construct nonunitary circuits that display a rich variety of entanglement scaling and phase transitions. One outcome of our approach is a nonunitary circuit for free fermions in one dimension that exhibits an entanglement transition from logarithmic scaling to volume-law scaling. This transition is accompanied by a "purification transition" analogous to that seen in hybrid projective-unitary circuits. We follow a similar strategy to construct a nonunitary two-dimensional (2D) Clifford circuit that shows a transition from area to volume-law entanglement scaling. Similarly, we spacetime rotate a 1D spin chain that hosts many-body localization to obtain a nonunitary circuit that exhibits an entanglement transition. Finally, we introduce an unconventional correlator and argue that if a unitary circuit hosts a many-body localization transition then the correlator is expected to be singular in its nonunitary counterpart as well.
\end{abstract}

DOI: 10.1103/PRXQuantum.2.040319

\section{INTRODUCTION}

Generic isolated quantum systems typically thermalize via the interaction between their constituents [1-5]. One exception to this is the phenomenon of many-body localization (MBL) [6-16] where strong disorder causes the system to develop signatures of nonergodicity such as subthermal entanglement under quantum quenches. More recently, it has been realized that new dynamical phases can also emerge in quantum systems subjected to projective measurements [17-48] due to the "quantum Zeno effect" [49]. Relatedly, one can consider evolution with more general nonunitary circuits [50-54], which typically exhibit nonergodic behavior as well. It is natural to wonder if there is any relation between these two classes of systems, namely, unitarily evolved systems that show singleparticle or many-body localization, and systems where nonunitarity plays a crucial role in suppressing ergodic behavior. In this work we explore such a connection using the idea of the spacetime rotation of a circuit [55-63].

*ts1015@ucsd.edu

Published by the American Physical Society under the terms of the Creative Commons Attribution 4.0 International license. Further distribution of this work must maintain attribution to the author(s) and the published article's title, journal citation, and DOI.
For a unitarily evolved system to exhibit localization, spatial disorder of course plays a central role. Evidence suggests that time translation invariance, whether continuous or discrete, is also crucial. For example, Floquet (i.e., time-periodic) circuits with spatial disorder can exhibit MBL phenomena [64-66], while unitary circuits that have randomness both in space and time tend to display ergodic behavior [67-74]. On the other hand, for the aforementioned nonunitary circuits displaying subthermal entanglement [17-48], translation invariance in the time or the space direction is not crucial. This is demonstrated by explicit construction of circuits consisting of projective measurements dispersed randomly in spacetime that host a transition from an area-law entanglement regime to a volume-law entanglement regime (see, e.g., Refs. [18-20]). A subclass of such nonunitary circuits has translation invariance in the space direction but lacks translation invariance in the time direction. Such circuits will be the focus of this work for reasons we discuss next.

The main idea we explore is the "spacetime rotation" of a quantum circuit [55-63] with a focus on unitary circuits that host a localization-delocalization transition. To set the stage, consider a general unitary circuit $U$ that acts for time $L_{t}$ on a $d$-dimensional system of spatial size $L_{1} \times L_{2} \times \cdots \times L_{d}$. From this, one can define a "partition function" $Z=\operatorname{tr}(U)$. Denoting the underlying degrees of freedom schematically by the symbol $\phi$, 


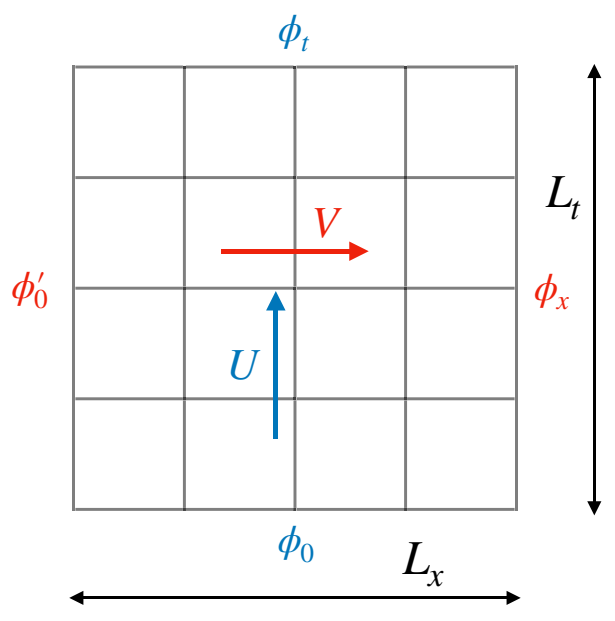

FIG. 1. The geometry of circuit rotation employed in this work illustrated for a 1D system. Given a unitary circuit $U$ that acts on a system of spatial size $L_{x}$, the wavefunction evolved for time $L_{t}$ is schematically given by $\left\langle\phi_{t}|U| \phi_{0}\right\rangle=\int D \phi(x, t) e^{i S(\phi)}$, where the fields labeled $\phi_{0}^{\prime}, \phi_{x}$ at the boundaries are also being integrated over in $D \phi(x, t)$, while $\phi_{0}, \phi_{t}$ act as boundary conditions. Using the same bulk action $S$, one may then define a rotated circuit $V$ that acts on a system with spatial extent $L_{t}$, such that the wavefunction at time $L_{x}$ is $\left\langle\phi_{x}|V| \phi_{0}^{\prime}\right\rangle=\int D \phi(x, t) e^{i S(\phi)}$. In this rotated circuit, the fields labeled $\phi_{0}, \phi_{t}$ are being integrated over in $D \phi(x, t)$, while $\phi_{0}^{\prime}, \phi_{x}$ act as boundary conditions.

one may represent $Z$ as a path integral in spacetime, $Z=\int D \phi(t,\{x\}) e^{i S[\phi]}, \quad$ where $\quad S[\phi(t,\{x\})]=\int d t d \vec{x} \mathcal{L}$ $(\phi, t, \vec{x})$ is the spacetime action and $\mathcal{L}\left(\phi, t, x_{1}, x_{2}, \ldots, x_{d}\right)$ is the corresponding Lagrangian. Let us now define a new Lagrangian $\tilde{\mathcal{L}}$ by interchanging $t$ and $x_{1}$ : $\tilde{\mathcal{L}}\left(\phi, t, x_{1}, x_{2}, \ldots, x_{d}\right)=\mathcal{L}\left(\phi, x_{1}, t, x_{2}, \ldots, x_{d}\right)$. For example, if $\mathcal{L}\left(\phi, t, x_{1}, x_{2}\right)=\left(\partial_{t} \phi\right)^{2}-\left[\left(\partial_{x_{1}} \phi\right)^{4}+\left(\partial_{x_{2}} \phi\right)^{6}+\phi^{4}\right]$ then $\tilde{\mathcal{L}}=-\left(\partial_{t} \phi\right)^{4}+\left(\partial_{x_{1}} \phi\right)^{2}-\left[\left(\partial_{x_{2}} \phi\right)^{6}+\phi^{4}\right]$. Since the original circuit $U$ is local, it implies that both $\mathcal{L}$ and $\tilde{\mathcal{L}}$ are also local. We use $\tilde{\mathcal{L}}$ to define a new "spacetime-rotated" circuit $V: \operatorname{tr}(V)=Z=\int D \phi(t,\{x\}) e^{i \int d t d \tilde{\mathcal{L}}}$. See Fig. 1 for an illustration, and Sec. II below for details. By design, the circuit $V$ acts for time $L_{1}$ on a system of spatial size $L_{t} \times$ $L_{2} \times \cdots \times L_{d}$. Crucially, $V$ is not guaranteed to be unitary [56]. This point was recently employed in Ref. [63] to design a method for emulating certain nonunitary circuits and their associated measurement-induced phase transitions without requiring extensive postselection. We note that in the context of imaginary time evolution, the idea of spacetime rotation to obtain a dual quantum Hamiltonian was first employed in Ref. [75].

In this work, we perform the aforementioned spacetime rotation on lattice models of Floquet circuits that are made out of unitaries with spatial disorder, and which display entanglement transitions due to the physics of localization. The rotated circuit $V$ will be generically nonunitary and, by construction, will possess translational invariance along a space direction, and disorder (randomness) along the time direction. A motivation for our study is that the rotated and unrotated circuits have the same partition function $Z$, which is closely related to the spectral form factor $[76,77]$ $\left(=|Z|^{2}\right)$. Since the spectral form factor in a Hamiltonian (or Floquet) system is expected to show singular behavior across a localization transition $[78,79]$, one may wonder if this fact has any consequence for the rotated circuit. In the special case when the rotation results in a unitary circuit, it was shown in Ref. [56] that the (unrotated) Floquet circuit is chaotic. Here we instead start from Floquet circuits that can be argued to display a localization transition (and therefore not always chaotic), and study the nonunitary circuits that result from their rotation.

The first example we study corresponds to a Floquet circuit that displays an Anderson localization transition due to quasiperiodic disorder. Rotating this circuit results in a one-dimensional (1D) free-fermion nonunitary circuit that exhibits a transition from a volume-law entanglement regime, $S \sim L$ ( $L$ is the spatial size), to a regime with entanglement characteristic of critical ground states: $S \sim \log (L)$. This is interesting because the known examples of nonunitary theories with free fermions have hitherto found only subextensive entanglement $[50-52,54]$. The fact that our nonunitary circuit is obtained from rotation of a unitary circuit plays a crucial role in its ability to support volume-law entanglement.

Next, we construct a 2D model where the unitary corresponds to a Floquet Clifford circuit and which displays a localization transition. Interestingly, spacetime rotating this circuit results in a nonunitary circuit consisting of only unitaries and "forced" projective measurements. We find that both the rotated and the unrotated circuits display an entanglement phase transition from a volume-law regime to an area-law regime.

The last example we study corresponds to a Floquet unitary circuit that displays a MBL transition [64-66]. The rotated, nonunitary counterpart again shows two distinct regimes, one where the entanglement scales as a volume law, and another where entanglement shows subextensive behavior.

Finally, we introduce an unconventional correlator that can be interpreted both within a unitary circuit and its nonunitary counterpart. We briefly discuss its measurement without employing any postselection. Using the ' $\ell$-bit' picture of MBL $[9,10]$, we provide a heuristic argument that this correlator exhibits singular behavior across a MBL transition.

The paper is organized as follows. In Sec. II, we provide a brief overview of the idea of spacetime rotating a circuit. In Sec. III, we discuss a Floquet model of noninteracting fermions in one dimension that displays a localizationdelocalization transition due to quasiperiodicity. We then study the phase diagram of the nonunitary circuit that results from its spacetime rotation. In Sec. IV we discuss 
a two-dimensional Clifford Floquet circuit that displays a localization-delocalization transition, and then study its spacetime-rotated version that turns out to be a hybrid circuit consisting of only unitaries and forced projective measurements. In Sec. V, we discuss a 1D interacting Floquet model that displays many-body localization transition and study the phase diagram of its rotated counterpart. In Sec. VI we introduce an unconventional correlator and discuss its physical consequences. Finally, in Sec. VII, we conclude with a discussion of our results.

\section{BRIEF OVERVIEW OF THE SPACETIME ROTATION OF A CIRCUIT}

Here we briefly review the idea of the spacetime rotation of a circuit using a 1D lattice model [56]. Although we specialize to one dimension for now, the discussion can be straightforwardly generalized to higher dimensions, as we do in Sec. IV. We begin by considering the following unitary Floquet circuit for a system of spatial size $L_{x}$ :

$$
U_{F}=e^{i \sum_{r=1}^{L_{x}} J_{X, r} X_{r}} e^{i \sum_{r=1}^{L_{x}} J_{Z, r} Z_{r} Z_{r+1}+i \sum_{r=1}^{L_{x}} h_{r} Z_{r}} .
$$

As discussed in the Introduction, a spacetime-rotated mapping is constructed by investigating the "partition function" $Z=\operatorname{tr}\left[\left(U_{F}\right)^{L_{t}}\right]$. Using the standard quantum-classical mapping, $Z$ can be expressed as a partition function of $L_{x} \times L_{t}$ classical variables $\left\{s_{r, t}\right\}$ in two dimensions with complex Gibbs weight: $Z \propto \sum_{\left\{s_{r, t}\right\}} e^{-S}$. Here the action $S$ reads

$$
-S=\sum_{r, t}\left(i \tilde{J}_{Z, r} s_{r, t} s_{r, t+1}+i J_{Z, r} s_{r, t} s_{r+1, t}+i h_{r} s_{r, t}\right) .
$$

The coupling between neighboring spins $\tilde{J}_{Z, r} s_{r, t} s_{r, t+1}$ along the time direction results from $e^{i J_{X},{ }_{r} X_{r}}$ in the Floquet unitary $U_{F}$, and the coupling constant $\tilde{J}_{Z, r}$ is determined as $\tilde{J}_{Z, r}=-\pi / 4+(i / 2) \log \left(\tan J_{X, r}\right)$. To obtain the spacetime-rotated circuit, one can now define a Hilbert space for $L_{t}$ spins on a given fixed-r timelike slice (Fig. 1). Correspondingly, the partition function can be written as $Z \propto \operatorname{tr}\left[\prod_{r=1}^{L_{x}} V_{r}\right]$, where $V_{r}$ acts on a Hilbert space of $L_{t}$ spins: $V_{r}=e^{i \tilde{J}_{X, r} \sum_{t} X_{t}} e^{i \tilde{J}_{Z, r} \sum_{t} Z_{t} Z_{t+1}+i h_{r} \sum_{t} Z_{t}}$ with $\tilde{J}_{X, r}=$ $\tan ^{-1}\left(-i e^{-2 i J_{Z, r}}\right)$. Altogether, $e^{i J_{X, r} X_{r}}$ in $U_{F}$ is mapped to $e^{i \tilde{J}_{Z, r} Z_{t} Z_{t+1}}$ in $V_{r}$, and $e^{i J_{Z, r} Z_{r} Z_{r+1}}$ in $U_{F}$ is mapped to $e^{i \tilde{J}_{X, r} X_{t}}$ in $V_{r}$.

Finally, by exchanging the labels of spacetime coordinates $r \leftrightarrow t$, one can construct the spacetime-rotated circuit $V(T)$ that evolves the system for a time $T$ and acts on a Hilbert space of size $L, V(T)=\prod_{t=1}^{T} V_{t}$, where

$$
V_{t}=e^{i \tilde{J}_{X}(t) \sum_{r=1}^{L} X_{r}} e^{i \tilde{J}_{Z}(t) \sum_{r=1}^{L} Z_{r} Z_{r+1}+i h(t) \sum_{r=1}^{L} Z_{r} .}
$$

A few remarks are in order. First, $V(T)$ has the space translational invariance resulting from the time translation invariance in the unrotated Floquet circuit $U_{F}$. Second, $V(T)$ is generically nonunitary except for the self-dual points $\left|J_{X, r}\right|=\left|J_{Z, r}\right|=\pi / 4$ [56]. Third, in the special case when $J_{X, r}, J_{Z, r}$, and $h_{r}$ are restricted to $\{0, \pm \pi / 4\}, V(T)$ corresponds to a hybrid quantum circuit with only unitary gates and forced projective measurements. While a $\pi / 4$ coupling gives unitary operation as just mentioned, $J_{X, r}=$ 0 implies that the spin at site $r$ is frozen in the unrotated circuit, and, hence, in the rotated circuit, this corresponds to a forced projective measurement of $\left(1+Z_{i} Z_{i+1}\right) / 2$ on two neighboring spins. Similarly, $J_{z, r}=0$ corresponds to a forced projective measurement of $(1+X) / 2$ on a single site. The fact that a forced projective measurement can arise from the spacetime rotation of a unitary gate has also been previously noted in Ref. [63]. Finally, once we have obtained the form of $V$, we let the corresponding system size $L$ and the evolution time $T$ [Eq. (3)] be free parameters that are independent of the system size and evolution time of the Floquet unitary $U_{F}$ from which it was obtained. That is, we do not impose the conditions $T=L_{x}, L=L_{t}$ when we compare various properties of $V$ with $U_{F}$.

Having reviewed the mapping between a unitary and its "spacetime dual," in the rest of the paper we consider several Floquet unitary circuits that exhibit entanglement transitions due to the physics of localization, and explore the phase diagrams of their spacetime duals.

\section{SPACETIME ROTATION AND ENTANGLEMENT TRANSITION IN A QUASIPERIODIC CIRCUIT}

As a first example, we consider a Floquet circuit in one space dimension hosting a localization-delocalization transition. We recall that models with quasiperiodic randomness, such as the Aubry-André-Harper (AAH) model [81-83], can evade Anderson localization [84] in one dimension. The AAH model is given by $H=$ $-t \sum_{r}\left(c_{r}^{\dagger} c_{r+1}+\right.$ H.c. $)-2 \lambda \sum_{r} \cos (2 \pi Q r+\delta) c_{r}^{\dagger} c_{r}$, where $c_{r}$ and $c_{r}^{\dagger}$ are the fermion creation and annihilation operators. When the on-site potential is incommensurate, i.e., the wavenumber $Q$ is irrational, all single-particle eigenstates are delocalized (localized) for $|t|>|\lambda|(|t|<|\lambda|)$ and arbitrary offset $\delta$. Motivated by this, we consider a Floquet circuit model with the unitary

$$
U_{F}=e^{i J \sum_{r} X_{r} X_{r+1}} e^{i \sum_{r} h_{r} Z_{r}}
$$

for a spin-1/2 chain of size $L$ with periodic boundary conditions. We choose $J=1$, and $h_{r}$ to be quasiperiodic: $h_{r}=$ $h+\lambda \cos (2 \pi Q r+\delta)$, where $Q$ is set to $2 /(1+\sqrt{5})$ (the inverse Golden ratio) and $h=2.5$. We note that Chandran and Laumann [85] studied the incommensurate AAH modulation in the transverse field Ising model, and found that, due to the interplay between symmetry and incommensurate modulation, it exhibits a rich phase diagram, including 
phases with delocalized, localized, and critical states that sometimes also break the Ising symmetry spontaneously.

Using the above Floquet unitary $U_{F}$, we construct the corresponding spacetime-rotated circuit $V$ as discussed above in Sec. II:

$$
V(T)=\prod_{t=1}^{T} e^{i \tilde{h} \sum_{r} Z_{r}} e^{i \sum_{r} \tilde{J}(t) X_{r} X_{r+1}}
$$

with $\tilde{J}(a)=-\pi / 4+(i / 2) \log \left(\tan h_{a}\right)$ and $\tilde{h}=\tan ^{-1}$ $\left(-i e^{-2 i J}\right)$. Note that the circuit $V$ is translationally invariant in space at each fixed time slice, but quasiperiodic in time.

Now we discuss the entanglement structure of longtime-evolved states $(T \gg L)$ from a product state $\left|\psi_{0}\right\rangle$ :

$$
|\psi(T)\rangle=\frac{U\left|\psi_{0}\right\rangle}{\sqrt{\left\langle\psi_{0}\left|U^{\dagger} U\right| \psi_{0}\right\rangle}} .
$$

Here $U$ is chosen as $\left(U_{F}\right)^{T}$ and $V(T)$ for the Floquet circuit and its spacetime dual, respectively. Using the Jordan-Wigner transformation, we map these circuits to a problem involving free fermions, and numerically compute the entanglement entropy using the correlation matrix technique [86-88] (see Appendix A 1 for the details).

For the unrotated circuit $U_{F}$, we find that the entanglement entropy exhibits a volume-law scaling for $\lambda \lesssim$ 0.64 and an area-law scaling for $\lambda \gtrsim 0.81$ [Fig. 2(a)]. In the intermediate regime, $0.64 \lesssim \lambda \lesssim 0.81$ [Fig. 2(b)], we find that $S_{A} \sim O\left(L^{\gamma}\right)$ with $0<\gamma<1$. Notably, deep in the volume-law phase, the entanglement entropy density $S_{A} / L_{A} \approx 0.386$ regardless of $\lambda$, which is very close to the average value predicted for random quadratic Hamiltonians of free fermions derived in Ref. [80]: $s_{r}=$ $\log 2-\left[1+f^{-1}(1-f) \log (1-f)\right] \approx 0.386$ at $f=1 / 2$. We also explore delocalization properties of the singleparticle eigenfuntions of the circuit $U_{F}$ in terms of free fermions and find three distinct phases (see Appendix A 2), in line with the late-time entanglement entropy studied here.

We now discuss the spacetime-rotated circuit $V$. We find that it also exhibits a transition in the entanglement entropy of long-time-evolved states. Figure 2(c) indicates that there is a transition in the entanglement entropy density $S_{A} / L_{A}$ at $\lambda \approx 0.64: S_{A}$ follows a volume law for $\lambda \lesssim 0.64$, and obeys a subvolume scaling for $\lambda \gtrsim 0.64$. We also note that in the volume-law regime, the coefficient of the volume law varies continuously, in strong contrast to the volumelaw phase of the unrotated unitary circuit. To elucidate the nature of the subvolume-law regime, we study $S_{A}$ versus $L$, and find that it scales logarithmically with the system size $L: S_{A} \sim \alpha \log (L)$ [see Fig. 2(d)] with $\alpha$ being a number that depends on $\lambda$. We also attempted a scaling collapse for the entanglement close to the critical point in the nonunitary circuit; see Appendix A 3. The collapse is reasonably good in the volume-law regime, while it does not work well in the subvolume-law regime. We suspect that this may be related to the fact that the coefficient $\alpha$ in the logarithmic scaling of entanglement varies continuously with $\lambda$.

A heuristic argument relates the physics of localization in the unitary circuit to the physics of the quantum Zeno effect [49] in the rotated nonunitary circuit, and also suggests that the aforementioned entanglement transition is likely to occur at $\lambda_{c}=\pi-h \approx 0.64$, in line with our numerical observations. For the unrotated circuit $U_{F}$, the condition $\lambda>\lambda_{c}$ implies that some $h_{r}$ in the term $e^{i h_{r} Z_{r}}$ is arbitrarily close to $\pi$. Mapping the spin chain to Majorana fermions using the Jordan-Wigner transition, the corresponding location $r$ then has a broken bond between two neighboring sites of the Majorana fermions, thereby impeding their propagation. In contrast, from the point of view of the rotated circuit $V$, the spacetime rotation of the term $e^{i h_{r} Z_{r}}$ at $h_{r}=\pi$ corresponds to the two-spin gate $e^{i \tilde{J} X_{j} X_{j+1}}$ with $\tilde{J}=-\pi / 4+(i / 2) \log \tan (\pi)$, which (a)

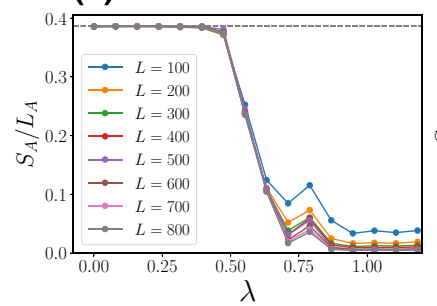

(b)

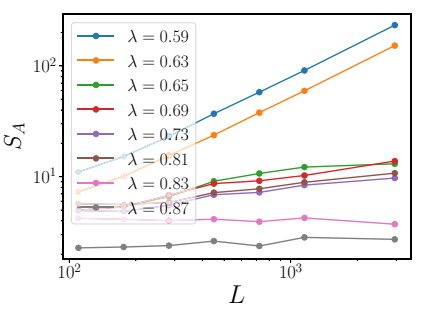

(c)

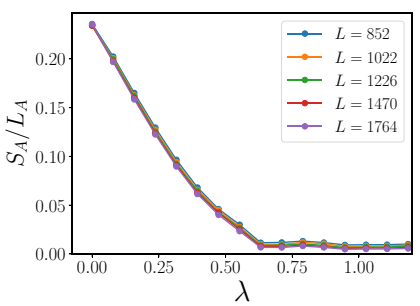

(d)

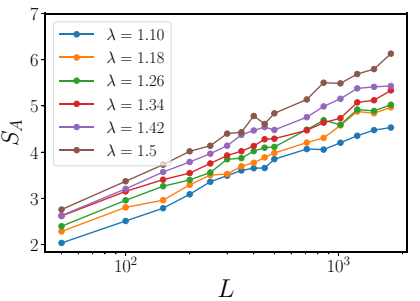

FIG. 2. Entanglement entropy $S_{A}$ of long-time-evolved states [evolution time up to $O\left(L^{2}\right)$ ] at the subsystem size fraction $L_{A} / L=$ 1/2. (a) Entanglement for the Floquet circuit, Eq. (4). The dashed gray line marks the entanglement entropy density averaged over all eigenstates of random quadratic Hamiltonians [80]. (b) Scaling of $S_{A}$ with $L$ in a narrower range of $\lambda$ for the same Floquet circuit as in (a). Here $S_{A}$ scales linearly with $L$ (i.e., volume law) for $\lambda \lesssim 0.64$ and follows an area law for $\lambda \gtrsim 0.81$. For $0.64 \lesssim \lambda \lesssim 0.81$, $S_{A} \sim O\left(L^{\gamma}\right)$ with $0<\gamma<1$. (c) Entanglement entropy density $S_{A} / L_{A}$ for the spacetime-rotated nonunitary circuit in Eq. (5). Here $S_{A} / L_{A}$ is nonzero for small modulation strength $\lambda$, while it vanishes for large $\lambda$. (d) Entanglement scaling in the regime $1.5 \gtrsim \lambda \gtrsim 1$ for the same circuit as in (c). One finds that $S_{A} \sim \log (L)$. 
therefore acts as a projector $\frac{1}{2}\left(1+X_{j} X_{j+1}\right)$. Crucially, such a two-site projection occurs uniformly in space (due to the space translational symmetry of the rotated circuit), leading to the absence of volume-law entanglement for time-evolved states.

Perhaps the most surprising aspect of our result is the presence of a volume-law phase since the previous works on nonunitary free-fermion circuits found phases only with subextensive entanglement [50-52,54]. For hybrid circuits consisting of unitary evolution interspersed with projective measurements, it was found in Ref. [17] that volume-law entanglement in a free-fermion chain is destroyed by the presence of arbitrarily weak measurement. Fidkowski et al. [46] argued for similar results. However, these results do not contradict ours since in the volume-law phase, our nonunitary circuit does not specifically correspond to unitary evolution interspersed with projective measurements but instead corresponds to more general evolution with a non-Hermitian Hamiltonian [see Eq. (5)].

To gain intuition for the origin of the volume-law phase, we consider a simplified circuit that has translation symmetry in both space and time, i.e., $V_{0}=$ $e^{i J \sum_{j} X_{j} X_{j+1}} e^{i h \sum_{j} Z_{j}}$, and allow $J$ and $h$ to be complex numbers. If $V_{0}$ is obtained from the spacetime rotation of a unitary circuit, a key feature is that the real parts of both $J$ and $h$ will be $\pi / 4$. Writing $J=\pi / 4+i \alpha_{J}$ and $h=\pi / 4+$ $i \alpha_{h}$, we find analytically that such a circuit leads to volumelaw entanglement at long times for any $\alpha_{J}$ and $\alpha_{h}$ (see Appendix B). The volume-law phase originates from the fact that, when $\operatorname{Re}(J)=\operatorname{Re}(h)=\pi / 4$, an extensive number of single-particle eigenvalues of the Floquet unitary are real. Setting $\alpha_{J}=\alpha_{h}=\alpha$, and using a simple quasiparticle picture [89], we find that the volume-law coefficient of entanglement decays exponentially with $\alpha: S_{A} / L_{A} \sim e^{-c \alpha}$ for $c>0$. Therefore, there is no area-law phase in this simplified, translationally invariant model. We numerically verified these results as well. Although we do not have similar analytical results for the circuit $V$ [Eq. (5)], we verified numerically that $\operatorname{Re}(\tilde{J})=\operatorname{Re}(\tilde{h})=\pi / 4$ (due to the circuit being obtained from the rotation of a unitary, namely $U_{F}$ ) is again essential to obtain a volume-law phase. In this sense, the volume-law phase of the nonunitary circuit is "symmetry protected" by the unitarity of the unrotated circuit.

One may also inquire about the role played by the time translation symmetry of the unitary circuit. If one chooses a different unitary circuit for each time slice then the localization is lost at any $\lambda$ and one only obtains a volume-law phase in the corresponding unitary circuit. We verified that the rotated circuit, which now lacks spatial translational symmetry, does not exhibit a phase transition. Therefore, at least for this specific problem, both the unitarity and the translation symmetry play a crucial role in obtaining the entanglement transition.

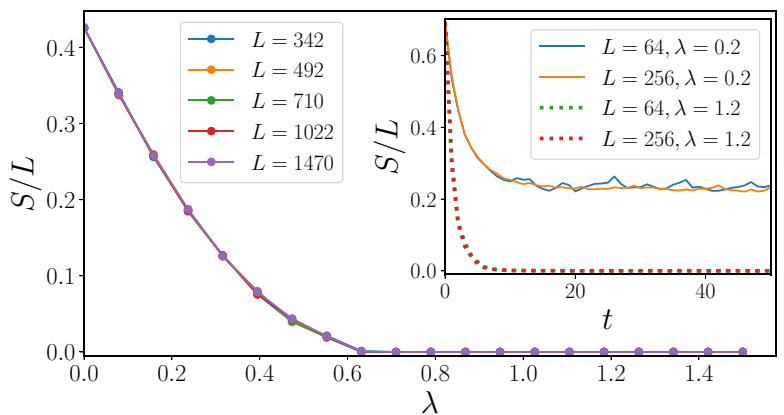

FIG. 3. The late-time entropy density $S / L$ for a density matrix that is initially in a completely mixed state [i.e., $\rho(t=0) \propto I$ ] and is evolved with the nonunitary circuit defined in Eq. (5). Inset: time evolution of $S / L$.

It was argued in Refs. [21,22] that a stable volumelaw entangled phase of pure states in a hybrid unitaryprojective circuit is a consequence of the robust errorcorrecting properties of the circuit against environmental monitoring. Consequently, a maximally mixed state $\rho=$ $I / 2^{L}$ evolved by the circuit will retain a finite residual entropy density up to an extremely long time, indicating stability against purification by monitoring. Motivated by these results, we studied the purification dynamics of a maximally mixed state evolved under our nonunitary circuit and investigated its von Neumann entropy density as a function of time. Remarkably, we found a sharp transition in the entropy density, where, for $\lambda<0.64$ (i.e., the volume-law entanglement phase), the system has a nonzero entropy density even at times $t \gg L$, and for $\lambda>$ 0.64 , the system is purified with a vanishing entropy density in a time that is independent of the system size $L$ (see Fig. 3 and Appendix A 4).

\section{SPACETIME ROTATION AND ENTANGLEMENT TRANSITION IN A 2D CLIFFORD CIRCUIT}

We next explore entanglement transitions in a twodimensional Floquet model and its spacetime dual. We consider the following Floquet unitary on a square lattice of size $L \times L$ :

$$
U_{F}=e^{-i(\pi / 4) \sum_{\langle i j\rangle} J_{i j} Z_{i} Z_{j}} e^{-i(\pi / 4) \sum_{i} h_{i} X_{i}} .
$$

Here each $J_{i j}, h_{i}$ is chosen to be 0 or 1 with probabilities $p$ and $1-p$, respectively. This is a Clifford circuit since it maps a Pauli string to another Pauli string: $e^{i(\pi / 4) Z_{1} Z_{2}} X_{j} e^{-i(\pi / 4) Z_{1} Z_{2}}=i Z_{1} Z_{2} X_{j}$ for $j=1,2$ and $e^{i(\pi / 4) X_{j}} Z_{j} e^{-i(\pi / 4) X_{j}}=i X_{j} Z_{j}$. Therefore, it can be efficiently simulated based on the Gottesman-Knill theorem [90-92]. The construction of the circuit $U_{F}$ is motivated from Ref. [93], although it differs from the precise circuit discussed in that work. 
To construct the spacetime-rotated circuit, we interchange the time coordinate $t$ and one of the space coordinates $x$ while leaving the other space coordinate $y$ unchanged. This results in the mapping as follows. Since $y$ coordinates are unchanged, the gate $e^{-i(\pi / 4) J_{i j} Z_{i} Z_{j}}$, with $\langle i j\rangle$ being a $y$-directed bond, is invariant under the spacetime-rotated mapping. The gate $e^{-i(\pi / 4) J_{i j} Z_{i} Z_{j}}$ along the $x$ direction in the unrotated circuit is mapped to a single-site gate $e^{i(\pi / 4) X},(1+X) / 2$ in the rotated circuit for $J_{i j}=1,0$, respectively. Finally, the single-site gate $e^{-i(\pi / 4) h_{i} X}$ in the unitary circuit maps to the two-site gate $e^{i(\pi / 4) Z_{i} Z_{j}},\left(1+Z_{i} Z_{j}\right) / 2$ on an $x$-directed bond in the nonunitary circuit for $h_{i}=1,0$, respectively. Therefore, the rotated circuit consists of unitary evolution interspersed with forced projective measurements, and is given by

$$
V(T)=\prod_{t=1}^{T}\left\{\prod_{y=1}^{L_{y}}\left[V_{X}(y, t) V_{Z Z, \mid}(y, t) V_{Z Z,-}(y, t)\right]\right\},
$$

where, for each $y$ and $t, V_{X}(y, t)=\prod_{x=1}^{L_{x}}\left(1+X_{x, y}\right) / 2$ or $\prod_{x=1}^{L_{x}} e^{i(\pi / 4) X_{x, y}} \quad$ with probabilities $p$ and $1-p$, $V_{Z Z, \mid}(y, t)=1$ or $\prod_{x=1}^{L_{x}} e^{i(\pi / 4) Z_{x, y} Z_{x, y+1}}$ with probabilities $p$ and $1-p$, and $V_{Z Z,-}(y)=\prod_{x=1}^{L_{x}}\left(1+Z_{x, y} Z_{x+1, y}\right) / 2$ or $\prod_{x=1}^{L_{x}} e^{i(\pi / 4) Z_{x, y} Z_{x+1, y}}$ with probabilities $p$ and $1-p$. Note that $V$ has translation symmetry along $x$ inherited from the time translation symmetry in the unrotated Floquet circuit.

Now we discuss the entanglement structure of longtime-evolved states. For both the unrotated and rotated circuits, we find an entanglement transition between a volume-law phase and an area-law phase at the same finite critical probability $p=p_{c} \approx 0.28$ (see Fig. 4). Assuming the scaling form of entanglement entropy $\left|S_{A}(p)-S_{A}\left(p_{c}\right)\right|=F\left[\left(p-p_{c}\right) L^{1 / \nu}\right]$, we find that the correlation length exponent $v$ however differs in the two circuits $(v \approx 0.38$ for the unrotated circuit and $v \approx 0.49$ for the rotated one). The coefficient of the volume-law entanglement varies continuously in both circuits and vanishes continuously across the phase transition.

We also analyzed entanglement scaling at the critical point, and found that both in the rotated and unrotated circuits, the data are indicative of the scaling $S \sim L \log L$, which is reminiscent of results in Refs. [34,40,51]; see Appendix C. However, as pointed out in Ref. [42], on small system sizes, a slight error in the location of the critical point can make an area-law scaling, $S \sim L$, appear as a $S \sim L \log L$ scaling. Therefore, one may need to study larger system sizes to be conclusive. As an aside, we note that the scaling form $S \sim L \log L$ is not allowed for a system described by a unitary, Lorentz invariant field theory at low energies due to the constraint $d^{2} S / d L^{2} \leq 0$ [94].

One may ask whether the time translation symmetry is crucial to obtain the observed transitions. Specifically, consider a circuit where independent unitaries of the form in Eq. (7) are applied at each time slice. In the (unrotated) unitary circuit, as one might expect, breaking time translational invariance always leads to volume-law entanglement [67-74]. We confirmed that rotating such a circuit leads to a hybrid projective-unitary circuit that also always exhibits a volume-law scaling. This is because the problem now essentially corresponds to anisotropic bond percolation in three dimensions where no bonds are removed along one of the directions (namely $y$ ) and are removed with probability $p$ along the other two directions ( $x$ and $t$ ). Such a model is known to not exhibit a percolation transition for any $p$ [95].

One may also consider the Floquet circuit [Eq. (7)] and its spacetime dual [Eq. (8)] in one dimension. In this case, however, both the unitary circuit and its rotated counterpart are in the area-law phase for any nonzero $p$. To see this, let us consider the unitary circuit and note that, when $p=0$, the spatial support of a single-site Pauli operator (a)

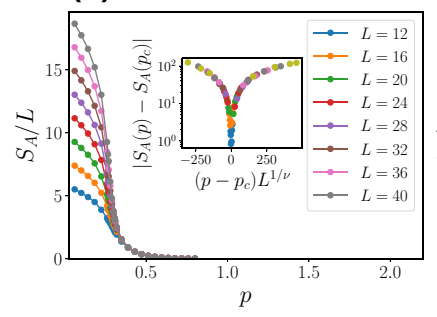

(b)

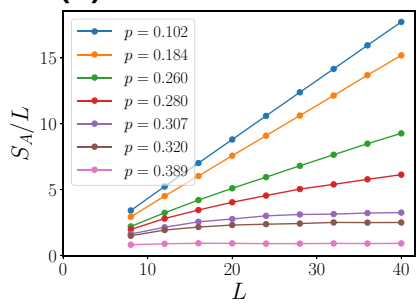

(c)

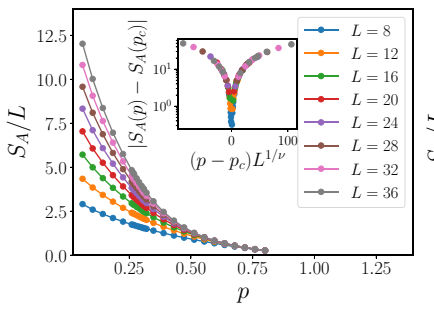

(d)

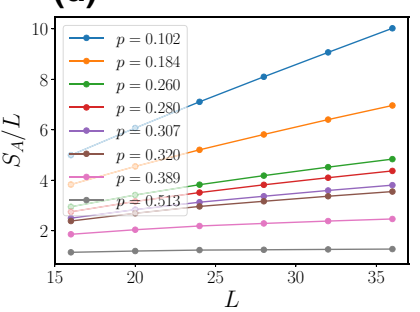

FIG. 4. (a) Long-time entanglement entropy $S_{A}$ of a subregion $A$ of size $L / 2 \times L$ averaged over $O\left(10^{3}\right)$ random realizations of the unitary circuit defined in Eq. (7) as a function of the probability $p$. The inset shows the scaling collapse across the critical point between a volume-law regime and an area-law regime with $\left(p_{c}, v\right) \approx(0.28,0.38)$. (b) $S_{A} / L$ for the same system as in (a) as a function of $L$ for various values of $p$. For small $p, S_{A} / L \sim L$, i.e., $S_{A} \sim L^{2}$, while for large values of $p, S_{A} / L$ is independent of $L$, signifying area-law scaling. (c) Long-time entanglement entropy of a subregion of size $L / 2 \times L$ averaged over $O\left(10^{4}\right)$ random realizations of the rotated nonunitary circuit [Eq. (8)]. The inset shows the scaling collapse with $\left(p_{c}, v\right) \approx(0.28,0.49)$. (d) Plot of $S_{A} / L$ for the same system as in (c) as a function of $L$ for various values of $p$. Again, for small $p, S_{A} / L \sim L$, i.e., $S_{A} \sim L^{2}$, while for large values of $p, S_{A} / L$ is independent of $L$. 
grows with time, leading to volume-law entanglement at long times. On the other hand, when $p \neq 0$, there is a finite density of locations [of $O(1 / p)$ ] where the $Z Z$ or the $X$ gates are absent. These locations impose a "wall" such that the end of a stabilizer string cannot grow beyond these walls. This leads to area-law entanglement $S_{A} \lesssim O(1 / p)$. In contrast, the $2 \mathrm{D}$ circuit discussed above allows for a volume-law phase for small nonzero $p$ since a local Pauli stabilizer spreads as a membrane that can bypass the points corresponding to the absent $Z Z$ or $X$ gates. Such a picture suggests that the entanglement transition may be related to a percolation transition, similar to Ref. [93]. However, the correlation length exponent we numerically obtain differs from the prediction of percolation in two dimensions. It would be worthwhile to revisit this question in more detail in the future.

\section{SPACETIME ROTATION OF AN INTERACTING FLOQUET MBL CIRCUIT}

Finally, we present numerical results on an interacting Floquet model of the form in Eq. (1), i.e.,

$$
U_{F}=e^{i J_{x} \sum_{r} X_{r}} e^{-i \tau \sum_{r} Z_{r} Z_{r+1}-i \tau \sum_{r} h_{r} Z_{r},}
$$

where $\tau=0.8$, and $h_{r}$ is a Gaussian random variable with mean $\bar{h}=0.8090$ and variance $W=1.421$. As shown in Ref. [66], tuning $J_{x}$ induces a transition from a MBL to an ergodic phase, where the Floquet eigenstates exhibit area-law entanglement for small $J_{x}$ and volume-law entanglement for large $J_{x}$. Here we study the corresponding spacetime dual nonunitary circuit.

As a benchmark, we first confirm the MBL-ergodic transition found in Ref. [66] for the Floquet unitary circuit. Using exact diagonalization (ED), we study the half-chain entanglement entropy $S_{A}$ averaged over all eigenstates of $U_{F}$, and average the data from 200 random realizations of $U_{F}$. We find clear signatures of a transition from a subextensive regime to a volume-law regime at finite $J_{x}=J_{x, c}$. Since eigenstates are localized for $J_{x}<J_{x, c}$ and are expected to resemble an infinite-temperature pure state [i.e., a random pure (Page) state [96] with entanglement entropy $\left.S_{R}=0.5(L \log 2-1)\right]$ for any $J_{x}>J_{x, c}$, we perform a data collapse, assuming the scaling form $S_{A} / S_{R}=$ $F\left[\left(J_{x}-J_{x, c}\right) L^{1 / \nu}\right]$, and find the critical point $J_{x, c} \approx 0.23$ with the correlation length exponent $v=1.09$ [Fig. 5(a) inset].

The spacetime-rotated circuit corresponding to $U_{F}$ is

$$
V(T)=\prod_{t=1}^{T} V_{t}, \quad V_{t}=e^{i \tilde{J}_{x} \sum_{r} X_{r}} e^{i \tilde{J}_{z} \sum_{r} Z_{r} Z_{r+1}-i \tau h(t) \sum_{r} Z_{r}},
$$

where the field $h$ is now random in the time direction due to the spacetime rotation, and the couplings $\tilde{J}_{x}, \tilde{J}_{z}$ are defined in Sec. II. We first analyze the entanglement structure of states evolved via $V$ for times $T \sim L$. We find signatures of a transition by tuning $J_{x}$ [see Fig. 5(b)]. In particular, when one plots entanglement entropy density, one finds a crossing at $J_{x} \approx 0.6$ [see Fig. $5(\mathrm{c})$ ], which separates a regime with volume-law entanglement from a regime where the entanglement is subextensive.

Finally, we study the entanglement dynamics of an ancilla qubit that is initially maximally entangled with the system, following the protocol in Refs. [21,22]. We evolve the system for time $T \sim L$, and find a crossing around $J_{x} \approx 0.4$ [see Fig. 5(d)]. In addition, the entanglement $S$ of the ancilla qubit shows distinct features on two sides of this crossing (see Appendix D for numerical data). For $J_{x} \lesssim 0.4$, the entanglement entropy of the ancilla qubit decays from its initial value $(=\log 2)$ exponentially with time, while for $J_{x} \gtrsim 0.4$, it remains at its initial value for a while (i.e., exhibits a 'plateau'), followed by an exponential decay. To quantify the plateau interval, we define a "purification time" $t_{p}$ as the time after which (a)

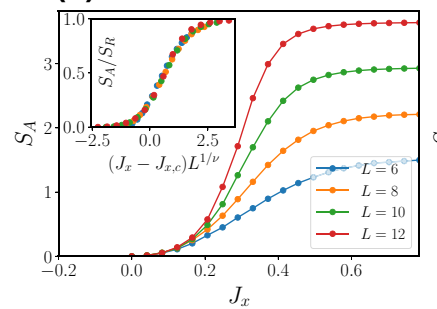

(b)

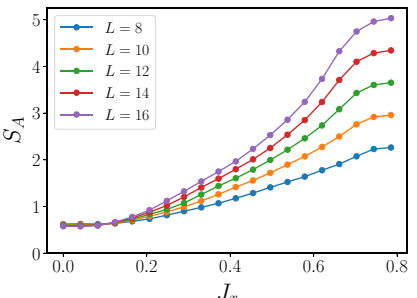

(c)

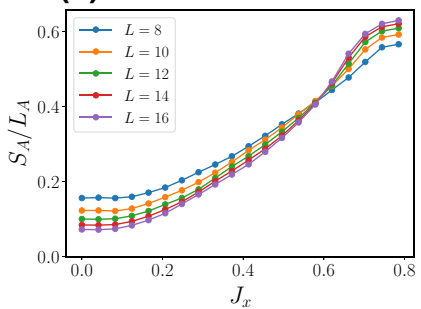

(d)

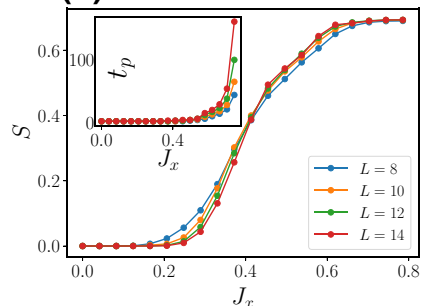

FIG. 5. (a) Half-chain entanglement entropy averaged over all eigenstates and 200 random realizations of the Floquet unitary $U_{F}$ defined in Eq. (9). The inset presents the data collapse based on the scaling ansatz: $S_{A} / S_{R}=F\left[\left(J_{x}-J_{x, c}\right) L^{1 / \nu}\right]$ with $\left(J_{x, c}, v\right)=$ $(0.23,1.09)$ and $S_{R}=0.5(L \log 2-1)$ being the entanglement entropy of a random pure state. (b) Entanglement entropy of longtime-evolved states averaged over 150 random realizations of the spacetime-rotated nonunitary circuit [Eq. (10)]. (c) Entanglement entropy density of the same circuit as in (b). (d) The entanglement entropy of an ancilla qubit that is initially prepared in the maximally entangled state with the system, and then evolved for time $t \sim L$ with the nonunitary circuit in Eq. (10). Averaging is done over 2000 realizations of the disorder. Inset: time scale $t_{p}$ that measures the persistence of the entanglement of the ancilla qubit. 
the entanglement of the ancilla qubit has dropped below 0.65 [approximately $0.94 \log (2)]$. We find that $t_{p} \approx O(1)$ for $J_{x} \lesssim 0.4$, while it increases with system size $L$ for $J_{x} \gtrsim$ 0.4 (Fig. 5 inset). Notably, for large enough $J_{x}(\gtrsim 0.7)$, we find that $t_{p}$ grows superlinearly with $L$, and therefore the nonunitary circuit may potentially serve as a good quantum error-correcting code.

Finally, we note that different values of the crossing points in different measures suggest that the finite size effects are likely strong at these system sizes. However, at the very least, the trends strongly indicate a stable volumelaw phase at $J_{x} \gtrsim 0.6$ [see Fig. 5(c)], and a phase with subextensive entanglement at small but nonzero $J_{x}$.

\section{SPACETIME-ROTATED CORRELATORS: POSTSELECTION-FREE MEASUREMENT AND PHYSICAL CONSEQUENCES}

Since the circuits related by spacetime rotation have the same bulk action $S$ (see the Introduction and Fig. 1), it is natural to seek a relation between their physical observables. At the outset, one notes that conventional correlation functions such as $\left\langle\psi_{0}\left|U^{\dagger} O U\right| \psi_{0}\right\rangle$ in the unitary circuit are not related to similarly defined correlations functions in its spacetime-rotated nonunitary $V$, such as $\left\langle\psi_{0}^{\prime}\left|V^{\dagger} O V\right| \psi_{0}^{\prime}\right\rangle /\left\langle\psi_{0}^{\prime}\left|V^{\dagger} V\right| \psi_{0}^{\prime}\right\rangle$. Referring to Fig. 1, this is because in the former case, the fields $\phi_{0}$ and $\phi_{t}$ are held fixed to define the wavefunction, and the fields $\phi_{0}^{\prime}$ and $\phi_{x}$ are being summed over, while in the latter case, it is the other way around. However, consider the following object (see Fig. 6):

$$
C\left(x_{1}, t_{1} ; x_{2}, t_{2}\right)=\frac{\int D \phi \phi_{1}\left(x_{1}, t_{1}\right) \phi_{2}\left(x_{2}, t_{2}\right) e^{i S(\{\phi\})}}{\int D \phi e^{i S(\{\phi\})}} .
$$

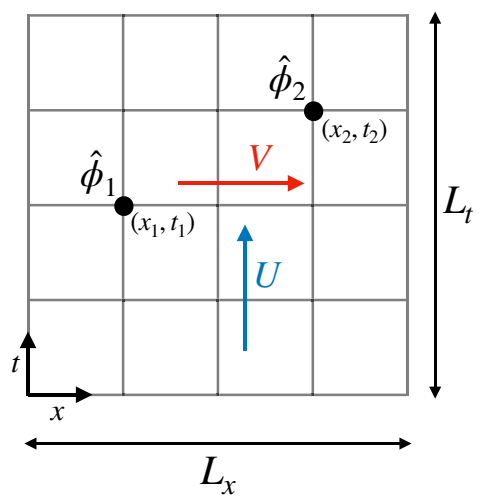

FIG. 6. Geometry for the correlation function $C\left(x_{1}, t_{1} ; x_{2}, t_{2}\right)$ defined in the main text [Eq. (11)]. It can be interpreted in two different ways: either as a correlation function for a system evolving unitarily with circuit $U$, or as a correlation function for a system evolving with the rotated nonunitary circuit $V$; see Eq. (12).
Since the action $S$ is invariant under spacetime rotation and one is summing over all fields in the above integral, $C$ has a well-defined meaning in both the rotated and unrotated circuits:

$$
\begin{aligned}
C\left(x_{1}, t_{1} ; x_{2}, t_{2}\right) & =\frac{\operatorname{tr}\left[U\left(t_{2}, t_{1}\right) \hat{\phi}_{1} U\left(t_{1}, t_{2}\right) \hat{\phi}_{2}\right]}{\operatorname{tr}\left[U\left(t_{2}, t_{1}\right) U\left(t_{1}, t_{2}\right)\right]} \\
& =\frac{\operatorname{tr}\left[V\left(x_{2}, x_{1}\right) \hat{\phi}_{1} V\left(x_{1}, x_{2}\right) \hat{\phi}_{2}\right]}{\operatorname{tr}\left[V\left(x_{2}, x_{1}\right) V\left(x_{1}, x_{2}\right)\right]} .
\end{aligned}
$$

Here $U(a, b)$ and $V(a, b)$ are evolution operators from time $a$ to $b$ when $a<b$, while when $a>b, U(a, b)=$ $U\left(a, L_{t}\right) U(0, b)$ and $V(a, b)=V\left(a, L_{x}\right) V(0, b)$ (see Fig. 6 for definitions of $\left.L_{t}, L_{x}\right) ; \hat{\phi}_{1}, \hat{\phi}_{2}$ are operators corresponding to the fields $\phi_{1}, \phi_{2}$ in Eq. (11), whose spacetime insertion locations are shown in Fig. 6.

The correlation functions in Eqs. (11) and (12) are rather unconventional since there is no "backward trajectory" as in the standard Keldysh expression [97,98] for conventional correlation functions such as $\left\langle\psi_{0}\left|U^{\dagger} O U\right| \psi_{0}\right\rangle$. To measure such correlators experimentally, one may employ the idea of a control qubit that generates two branches of a many-body state [99-101]. For example, to measure $\left\langle\psi_{0}\left|U_{1} \hat{\phi}_{1} U_{2} \hat{\phi}_{2}\right| \psi_{0}\right\rangle$ for some $U_{1}, U_{2}$ and a product state $\left|\psi_{0}\right\rangle$, the total system is initially prepared in a state $\left|\psi_{0}\right\rangle \otimes(|\uparrow\rangle+|\downarrow\rangle)$, where the expression after " $\otimes$ " denotes the state of the control qubit. Using standard techniques [99-101], one then applies the operator $U_{2} \hat{\phi}_{2}$ on the "up branch" of this initial state, i.e., the state $\left|\psi_{0}\right\rangle \otimes|\uparrow\rangle$, and similarly, one applies the operator $\hat{\phi}_{1}^{\dagger} U_{1}^{\dagger}$ on the down branch. Finally, one measures the expectation value of the $\sigma^{x}$ and the $\sigma^{y}$ operators that act on the control qubit, which yields the object of interest, namely, the real and imaginary parts of $\left\langle\psi_{0}\left|U_{1} \hat{\phi}_{1} U_{2} \hat{\phi}_{2}\right| \psi_{0}\right\rangle$. The trace in Eq. (12) would then need to be approximated by sampling over several such expressions, although even a single or few such expressions may sometime capture the qualitative aspects of interest (see below).

Because of the unconventional nature of the correlator $C$, it is not obvious if it captures universal physics. We now provide a heuristic argument that $C$ is singular across the MBL transition. We recall that a MBL system hosts emergent " $\ell$-bit" degrees of freedom $[9,10]\left\{\tau^{z}\right\}$ that have a nonzero overlap with the local $\sigma^{z}$ operators, i.e., $\sigma_{i}^{z}=$ $Z \tau_{i}^{z}+\cdots$, where $Z \neq 0$ denotes the overlap between $\sigma_{i}^{z}$ and $\tau_{i}^{z}$ ("quasiparticle residue"). In particular, this implies that at long times $\sigma_{i}^{z}(t)=Z \sigma_{i}^{z}(0)+\cdots$, i.e., $\sigma_{i}^{z}(t)$ has a nonzero overlap with $\sigma_{i}^{z}(0)$. Let us use this fact to simplify the numerator of $C$ in Eq. (12): $\operatorname{tr}\left[U(0, T / 2) \sigma_{r}^{z} U(T / 2,0)\right.$ $\left.\sigma_{r}^{z}\right]=\operatorname{tr}\left[\sigma_{r}^{z}(T / 2) U(0, T / 2) U(T / 2,0) \sigma_{r}^{z}\right]=Z \operatorname{tr}[U(0, T / 2)$ $U(T / 2,0)]+\cdots$ with the three center dots denoting terms that are expected to vanish at long times after averaging over time and disorder. This suggests that in a MBL phase, $C(r, 0 ; r, T / 2)$ simply equals $Z$ at large $T$, 


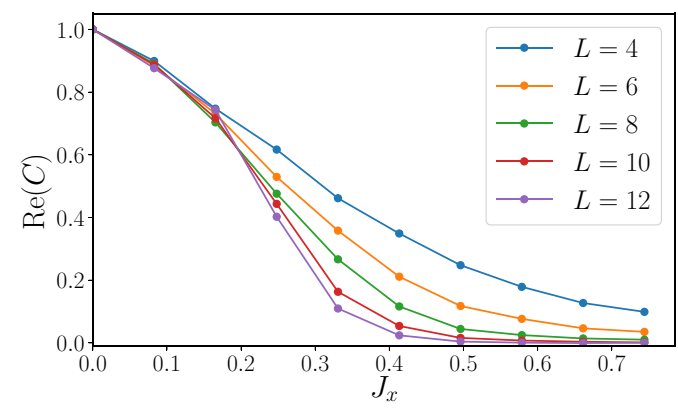

FIG. 7. Correlation function $C(r, 0 ; r, T / 2)$ for $T \gtrsim O(L)$ in the circuit $U_{F}$ defined in Eq. (9). The data presented are obtained by averaging over 2560 random realizations of $U_{F}$ for $L=$ $4,6,8,10$, and 512 random realizations of $U_{F}$ for $L=12$.

and therefore vanishes as one approaches the delocalization transition. We verified this expectation numerically using ED for the Floquet model studied in Sec. V; see Fig. 7. We also calculated a simpler correlator, namely, $\left\langle\psi_{0}\left|U(0, T / 2) \sigma_{r}^{z} U(T / 2,0) \sigma_{r}^{z}\right| \psi_{0}\right\rangle$, where $\left|\psi_{0}\right\rangle$ is a product state, and found that it behaves quite similarly to $C$.

We also studied the correlator in the 1D free-fermion circuit discussed in Sec. III as well as the 2D Clifford circuit discussed in Sec. IV. We found that the correlator fails to distinguish between the localized phase and the delocalized phase in either of these circuits for distinct reasons. For a localized free-fermion circuit, the time-evolved operator $\sigma_{i}^{z}(t)$ continues to have a nonzero overlap $Z$ with $\sigma_{i}^{z}$ at arbitrarily long times, i.e., $\sigma_{i}^{z}(t)=Z \sigma_{i}+\cdots$. However, due to the lack of dephasing in free-fermion circuits (see, e.g., Ref. [102]), the terms under the three center dots do not vanish even at long times despite averaging over disorder, and their contribution fluctuates in time significantly at all times. Consequently, the spacetime-rotated correlator does not provide a clear signature across the localization transition.

On the other hand, for a Clifford circuit, the correlator $C$ does not differentiate between a localized phase and a delocalized phase due to the absence of the " $\ell$-bit" picture $\sigma_{i}^{z}(t)=Z \sigma_{i}^{z}+\cdots$. Specifically, $\sigma_{i}^{z}(t)$ will always be a single product of Pauli operators over various sites, and the localization (delocalization) phase manifests in the bounded (un-bounded) spatial support of $\sigma_{i}^{z}(t)$, instead of the relative weight of various operators. Therefore, our aforementioned argument in the context of generic MBL systems does not apply.

We note that Ippoliti and Khemani [63] discussed an alternative method to relate quantities between a unitary circuit and its rotated nonunitary counterpart. In particular, Ippoliti and Khemani [63] considered a protocol where the purification dynamics in the nonunitary circuit can be obtained by a combination of unitary dynamics and projective measurements.

\section{SUMMARY AND DISCUSSION}

In this work, we employ the idea of the spacetime rotation of unitary circuits to construct nonunitary circuits that display entanglement phase transitions. We focus on specific Floquet unitary circuits that display localizationdelocalization transitions of various kinds (free fermion, Clifford, many body). We find that the delocalized (localized) regime of the unitary circuit maps to a regime with volume-law (area-law or critical) entanglement in the corresponding nonunitary circuit. Therefore, the spacetime rotation maps the physics of localization to the physics of the quantum Zeno effect. We also find that the entanglement transitions in the nonunitary circuits are accompanied by purification transitions of the kind introduced in Refs. [21,22]. We introduce an unconventional correlator in the nonunitary theory that can in principle be measured without requiring any postselection, and provide a heuristic argument that this correlator is singular across a MBL transition.

Our procedure leads to the construction of a nonunitary free-fermion circuit that supports volume-law entanglement, which has hitherto been elusive [50-52,54]. As discussed in Sec. III, we find that a nonunitary circuit obtained by the rotation of a free-fermion unitary circuit has the special property that the real parts of certain hopping elements are automatically pinned to $\pi / 4$. This leads to volume-law entanglement when the nonunitary circuit has translational symmetry in both space and time, and the possibility of a volume-law to area-law transition when disorder is introduced in the nonunitary circuit along the time direction.

Given our results, it is natural to ask if the spacetime rotation of a unitary circuit $U$ hosting a localizationdelocalization transition always leads to a nonunitary circuit $V$ that also shows an entanglement transition. Firstly, we note that a localization-delocalization transition in a unitary system will induce a singularity in the spectral form factor since the spectral form factor is well known to be sensitive to quantum chaos. Because of our mapping, the spectral form factor for the nonunitary theory will also be singular across the transition ( since $=|\operatorname{tr} U|^{2}=|\operatorname{tr} V|^{2}$ ). Recent progress [103] shows that, at least for a class of nonunitary evolution, the spectral form factor continues to encode features of quantum chaos. Furthermore, as discussed in Sec. VI, a correlator that is well defined in both the unitary and the nonunitary theory can be argued to be singular across a MBL transition. However, this correlator is a bit hard to interpret physically within the nonunitary theory. It will be worthwhile to pursue a physical understanding of the spectral form factor and the correlator in Sec. VI for the nonunitary theories studied in this paper.

We also explore the role played by the time translation symmetry of the unitary circuit. In the examples we study, breaking of time translation symmetry also leads to the 
absence of entanglement transition in the rotated nonunitary circuit. We suspect that the entanglement transitions in nonunitary circuits that are spacetime dual of time translationally invariant unitary circuits belong to a different universality class compared to those hosted by nonunitary circuits where such a symmetry is absent.

As argued in Ref. [63], if a nonunitary circuit is related to a unitary circuit via spacetime rotation then at least some of its properties (such as the purification rate) may be obtained purely via unitary evolution combined with a small number of projective measurements. Furthermore, as discussed in Sec. VI, an unconventional correlator in the nonunitary theory can be measured using only unitary operations. Applying these results to the examples discussed in this work would potentially allow one to access the physics of entanglement transitions in hybrid projective-unitary circuits without postselection.

We note that Jian et al. [52] introduced an interesting relation between nonunitary circuits of free fermions in $d+1$ spacetime dimensions and the Anderson localization-delocalization transition for Hermitian Hamiltonians in $d+1$ space dimensions. The basic idea employed is to relate the circuit in $d+1$ spacetime dimensions to the scattering matrix that describes the ChalkerCoddington model [104] in $(d+1)$-dimensional space. In contrast, our work focuses on relating a unitary and a nonunitary system that live in the same number of spacetime dimensions. It might be worthwhile to understand the volume-law phase in our nonunitary circuit of free fermions (Sec. III) and its higher-dimensional generalizations from the perspective in Ref. [52].

\section{ACKNOWLEDGMENTS}

We are grateful to John McGreevy and Yahya Alavirad for illuminating discussions and helpful comments on the draft. We thank Matteo Ippoliti, Tibor Rakovszky, and Vedika Khemani for pointing out an incorrect statement in the "Summary and Discussion" section of the first version of this preprint; see footnote [106] for details. T.G. acknowledges support from the National Science Foundation, under Grant No. DMR-1752417, and by an Alfred P. Sloan Research Fellowship. This work used the Extreme Science and Engineering Discovery Environment (XSEDE) [107], which is supported by the National Science Foundation Grant No. ACI-1548562. We acknowledge support from the University of California's Multicampus Research Programs and Initiatives (MRP-19601445).

Note added.-After the completion of this work, we became aware of a related work [105] (appearing in the same arXiv posting) that also considers entanglement dynamics in spacetime duals of unitary circuits. Our works are largely complementary and agree where they overlap.

\section{APPENDIX A: ADDITIONAL DETAILS ON 1 + 1D FLOQUET QUASIPERIODIC CIRCUIT}

\section{Entanglement entropy}

Here we outline the calculation of entanglement entropy of time-evolved states in the $1+1 \mathrm{D}$ circuit [Eq. (4)]

$$
U_{F}=e^{i J \sum_{j} X_{j} X_{j+1}} e^{i \sum_{j} h_{j} Z_{j}},
$$

where $h_{j}=h+\lambda \cos (2 \pi Q j+\delta)$ and $Q=2 /(1+\sqrt{5})$.

We first map the circuit to a fermionic model using the Jordan-Wigner transformation:

$$
Z_{i}=1-2 c_{i}^{\dagger} c_{i}, \quad c_{i}^{+}=\left(\prod_{j=1}^{i-1} Z_{j}\right) \sigma_{i}^{-}, \quad c_{i}^{-}=\left(\prod_{j=1}^{i-1} Z_{j}\right) \sigma_{i}^{+} .
$$

Correspondingly, $\quad X_{i}=\sigma_{i}^{-}+\sigma_{i}^{+}=\left[\prod_{j=1}^{i-1}\left(1-2 c_{j}^{\dagger} c_{j}\right)\right]$ $\left(c_{i}+c_{i}^{+}\right)$, and

$$
\begin{aligned}
\sum_{i=1}^{L} X_{i} X_{i+1}= & \sum_{i=1}^{L-1}\left(c_{i}^{\dagger}-c_{i}\right)\left(c_{i+1}^{\dagger}+c_{i+1}\right) \\
& -e^{i \pi N}\left(c_{L}^{\dagger}-c_{L}\right)\left(c_{1}^{\dagger}+c_{1}\right) \\
= & \sum_{i=1}^{L}\left(c_{i}^{\dagger}-c_{i}\right)\left(c_{i+1}^{\dagger}+c_{i+1}\right),
\end{aligned}
$$

where $e^{i \pi N}$ measures the total fermion number parity: $e^{i \pi N}=e^{i \pi \sum_{i} c_{i}^{\dagger} c_{i}}=\prod_{i=1}^{L}\left(1-2 c_{i}^{\dagger} c_{i}\right)$. We impose the antiperiodic boundary condition $c_{L+1}=-c_{1}$ for the even fermion parity sector and the periodic boundary condition $c_{L+1}=c_{1}$ for the odd fermion parity sector.

Since the Floquet dynamics does not conserve the total fermion number, it is more convenient to employ the Majorana fermions by defining $a_{2 j-1}=c_{j}+c_{j}^{\dagger}$ and $a_{2 j}=i\left(c_{j}-c_{j}^{\dagger}\right)$, which satisfy $\left\{a_{i}, a_{j}\right\}=2 \delta_{i j}$. The Floquet unitary defined in Eq. (A1) then reads

$$
U_{F}=U_{X X} U_{Z}=e^{-J \sum_{j=1}^{L} a_{2 j} a_{2 j+1}} e^{\sum_{j=1}^{L} h_{j} a_{2 j-1} a_{2 j}} .
$$

Since $U_{F}$ is Gaussian in Majorana fermions, the Majoranas evolve under $U_{F}$ as

$$
U_{F}^{\dagger} a_{i} U_{F}=U_{Z}^{\dagger} U_{X X}^{\dagger} a_{i} U_{X X} U_{Z}=\sum_{k} O_{i k} a_{k}
$$

where $O$ is an orthogonal matrix. Correspondingly, the Majoranas at time $t$ can be obtained by repeatedly applying the orthogonal transformation on $\left\{a_{i}\right\}: a_{i}(t)=\sum_{j}\left(O^{t}\right)_{i j} a_{j}$. Using this formalism, we can calculate the correlation matrix at time $t$, i.e., $\Gamma_{i j}(t)=\left\langle a_{i}(t) a_{j}(t)\right\rangle-\delta_{i j}$, from which 

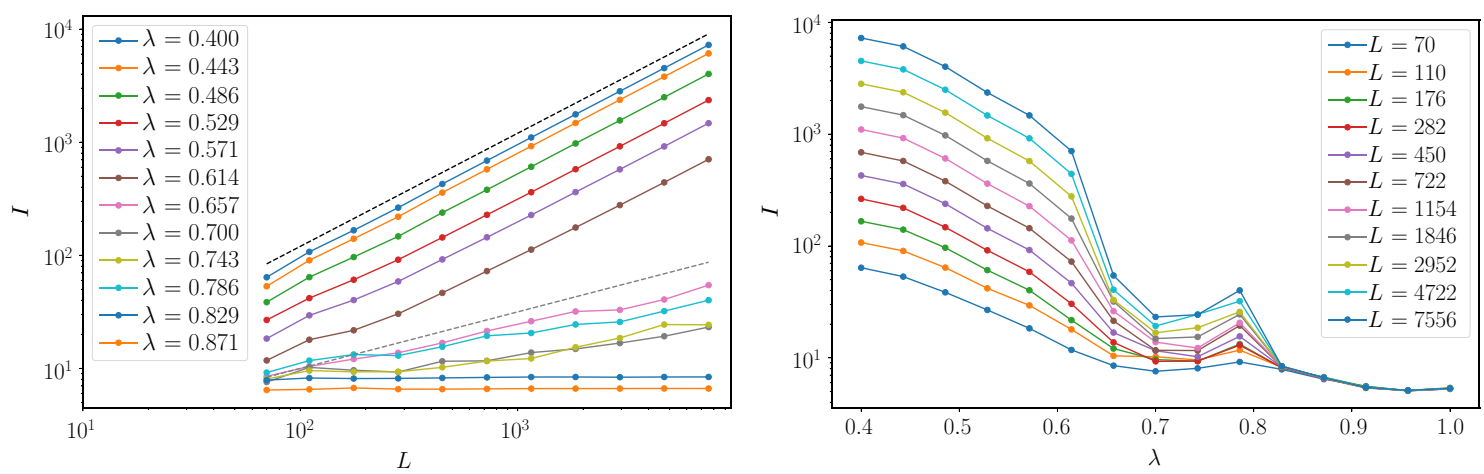

FIG. 8. Inverse participation ratio $(I)$ averaged over all eigenstates of the orthogonal matrix $O$ that governs the dynamics of Majorana fermions up to the total system size $L=7556$. In the left panel, the black and gray dashed lines serve as references for the scaling laws $I \sim L$ and $I \sim \sqrt{L}$, respectively.

the entanglement entropy between a region $A$ and its compliment can be found by diagonalizing $\Gamma_{A}(t)$, the restriction of the correlation matrix to the region $A$ (Refs. [86-88]):

$$
S_{A}=-\sum_{i=1}^{L_{A}}\left[\frac{1-v_{i}}{2} \log \left(\frac{1-v_{i}}{2}\right)+\frac{1+v_{i}}{2} \log \left(\frac{1+v_{i}}{2}\right)\right]
$$

with $\left\{ \pm v_{i}\right\}$ being the $2 L_{A}$ eigenvalues of $\Gamma_{A}(t)$.

\section{Single-particle eigenfunctions of the Floquet unitary}

Here we discuss the properties of the single-particle eigenfunctions of $U_{F}$ in terms of the Majorana fermions. We find signatures of three distinct phases, in line with the results from entanglement entropy of long-time-evolved states (Sec. III in the main text). Specifically, we study the inverse participation ratio $(I)$

$$
I=\frac{1}{\sum_{i}\left|\psi_{i}\right|^{4}}
$$

where $\psi_{i}$ is an eigenfunction of $O$ at the $i$ th Majorana site. We recall that the $I$ is a conventional tool to quantify the localization-delocalization property of wavefunctions. In one spatial dimension, an extended (delocalized) wavefunction has $\left|\psi_{i}\right| \sim O(1 / \sqrt{L})$, implying that $I \sim O(L)$. On the other hand, a localized wavefunction is mainly supported on a finite number of lattice sites, yielding $I \sim$ $O(1)$. Here we study the $I$ averaged over all eigenstates of $O$, and find that the averaged $I$ (denoted as $\langle I\rangle$ ) exhibits three different scalings with the system size $L$ as the modulation strength $\lambda$ is varied, similar to the entanglement entropy of long-time-evolved many-body states. For small $\lambda,\langle I\rangle$ scales as $O(L)$, a signature of a delocalized phase, while for large $\lambda,\langle I\rangle \sim O(1)$, corresponding to a localized phase. In addition, there is an intermediate regime
$(0.64 \lesssim \lambda \lesssim 0.8)$, where $\langle I\rangle$ scales as $O\left(L^{\gamma}\right)$ with $\gamma \sim 0.5$ (see Fig. 8).

\section{Scaling collapse of entanglement entropy}

Here we provide numerical data for the scaling collapse of the late-time entanglement entropy for the rotated circuit [Eq. (5)] (Fig. 9).

\section{Purification dynamics}

Here we present additional numerical results on the purification dynamics of a density matrix that is initially in a completely mixed state [i.e., $\rho(t=0) \propto I$ ] and is evolved with the nonunitary circuit defined in Eq. (5) (see Fig. 10). At $\lambda=0.2$ (i.e., in the volume-law phase), the entropy density $S / L$ decreases at short times and remains nonzero for the longest observed time $\left(t \sim 2^{L}\right)$. At $\lambda=1.2$ (i.e., in the critical phase), the entropy density decreases exponentially to zero within a characteristic time scale that is independent of $L$.

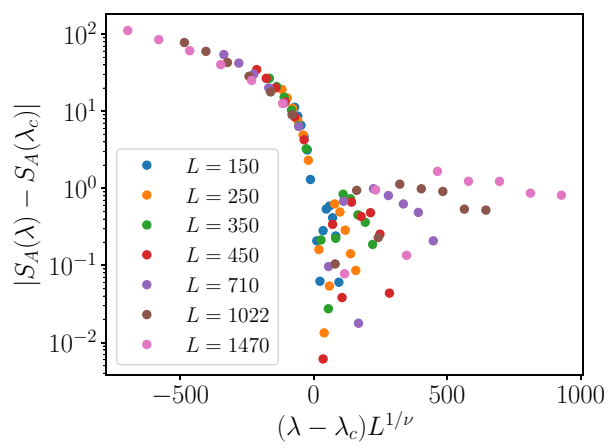

FIG. 9. Scaling collapse of the late-time entanglement entropy for the rotated circuit defined in Eq. (5). We use the scaling ansatz $\left|S_{A}(\lambda)-S_{A}\left(\lambda_{c}\right)\right|=F\left[\left(\lambda-\lambda_{c}\right) L^{1 / \nu}\right]$, where $\left(\lambda_{c}, v\right) \approx$ $(0.64,1.0)$ 

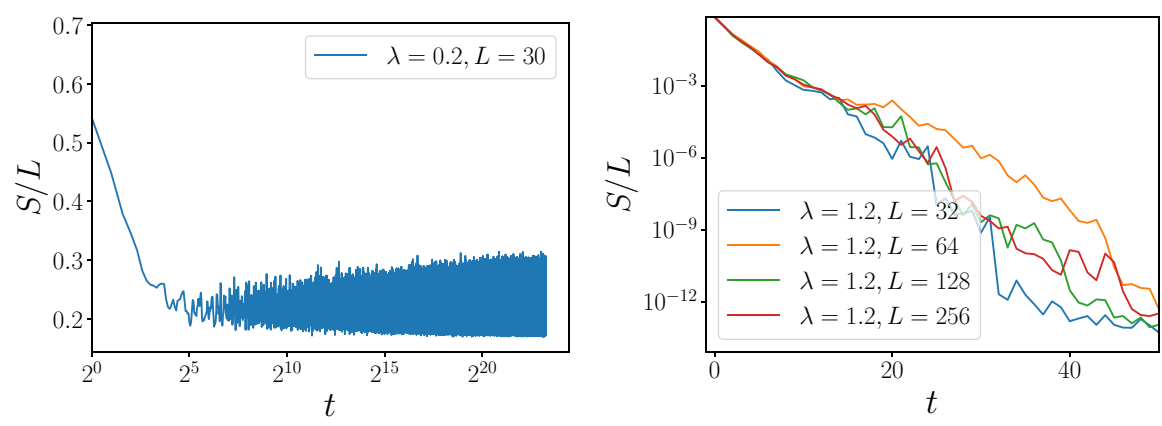

FIG. 10. Time evolution of entropy density for the nonunitary circuit defined in Eq. (5). The density matrix is prepared at $t=0$ in a completely mixed state, $\rho(t=0) \propto I$.

\section{APPENDIX B: 1 + 1D NONUNITARY CIRCUITS WITH SPACETIME TRANSLATIONAL INVARIANCE}

Given the Floquet unitary

$$
U_{F}=e^{i J \sum_{j} X_{j} X_{j+1}} e^{i h \sum_{j} Z_{j}},
$$

we show that, when the real parts of both $J$ and $h$ are $\pi / 4$, a time-evolved state at a typical long time exhibits volume-law entanglement. We solve the model using the standard Jordan-Wigner transformation, where we first introduce the complex fermions $c_{j}$ in real space, and then Fourier transform the fermions to momentum space $c_{k}=$ $(1 / \sqrt{L}) \sum_{j} e^{-i k j} c_{j}$. It follows that $U_{F}$ can be written as

$$
\begin{aligned}
U_{F}= & \prod_{k>0} \exp \left\{2 i J\left(\begin{array}{cc}
c_{k}^{\dagger} & c_{-k}
\end{array}\right)\left(\begin{array}{cc}
\cos k & i \sin k \\
-i \sin k & -\cos k
\end{array}\right)\left(\begin{array}{c}
c_{k} \\
c_{-k}^{\dagger}
\end{array}\right)\right\} \\
& \times \exp \left\{-2 i h\left(c_{k}^{\dagger} c_{k}+c_{-k}^{\dagger} c_{-k}\right)\right\} .
\end{aligned}
$$

By introducing the Majorana fermions $a_{k}=c_{k}+c_{k}^{\dagger}, b_{k}=$ $i\left(c_{k}-c_{k}^{\dagger}\right), U_{F}$ reads

$$
\begin{aligned}
U_{F}= & \prod_{k>0} e^{J\left[\cos k\left(a_{k} b_{k}+a_{-k} b_{-k}\right)-\sin k\left(a_{k} a_{-k}-b_{k} b_{-k}\right)\right]} \\
& \times e^{-h\left(a_{k} b_{k}+a_{-k} b_{-k}\right)}
\end{aligned}
$$

Defining $A_{k}=\left(a_{k}, b_{k}, a_{-k}, b_{-k}\right)^{T}$, one finds that $U_{F}=$ $\prod_{k>0} e^{A_{k}^{T} W_{X X, k} A_{k} / 4} e^{A_{k}^{T} W_{Z, k} A_{k} / 4}$, where

$$
\begin{aligned}
W_{X X, k} & =\left(\begin{array}{cccc}
0 & 2 J \cos k & -2 J \sin k & 0 \\
-2 J \cos k & 0 & 0 & 2 J \sin k \\
2 J \sin k & 0 & 0 & 2 J \cos k \\
0 & -2 J \sin k & -2 J \cos k & 0
\end{array}\right), \\
W_{Z, k} & =\left(\begin{array}{cccc}
0 & -2 h & 0 & 0 \\
2 h & 0 & 0 & 0 \\
0 & 0 & 0 & -2 h \\
0 & 0 & 2 h & 0
\end{array}\right) .
\end{aligned}
$$

Since the product of two Gaussian states remains a Gaussian, $U_{F}$ can be simplified as

$$
U_{F}=\prod_{k>0} e^{A_{k}^{T} W_{k} A_{k} / 4},
$$

where

$$
e^{W_{k}}=e^{W_{X X}, k} e^{W_{Z}, k}
$$

One can introduce a Floquet Hamiltonian $-i H_{k}=$ $A_{k}^{T} W_{k} A_{k} / 4$ so that $U_{F}=\prod_{k>0} e^{-i H_{k}}$. Being quadratic in Majoranas, $H_{k}$ can be diagonalized using an orthogonal transformation on $A_{k}$ as $H_{k}=(i / 2) \varepsilon_{k}\left(\gamma_{k}^{\prime} \gamma_{k}^{\prime \prime}+\gamma_{-k}^{\prime} \gamma_{-k}^{\prime \prime}\right)$, where $\varepsilon_{k}$ is the corresponding energy. In particular, $\varepsilon_{k}$ can be obtained from $w_{k}$ (eigenvalues of $W_{k}$ ) through

$$
\varepsilon_{k}= \pm \sqrt{-w_{k}^{2}} .
$$

After some algebra, one finds the eigenvalues of $e^{W_{k}}$ :

$$
e^{w_{k}}=\frac{x}{4} \pm \sqrt{\left(\frac{x}{4}\right)^{2}-1}
$$

with $\quad x=2(1+\cos k) \cos (2 h-2 J)+2(1-\cos k) \cos$ $(2 h+2 J)$. Below we find that the number of $k$ modes with purely real energy $\varepsilon_{k}$ is extensive in the system size $L$ when the real parts of both $J$ and $h$ are $\pi / 4$, which is ultimately responsible for the volume-law bipartite entanglement of long-time-evolved states. To analyze this case, we take $h=\pi / 4+i \alpha_{h}$ and $J=\pi / 4+i \alpha_{J}$, where $\alpha_{h}, \alpha_{J}$ are real, and find that

$$
\begin{aligned}
x= & 2(1+\cos k) \cosh \left(2 \alpha_{h}-2 \alpha_{J}\right) \\
& -2(1-\cos k) \cosh \left(2 \alpha_{h}+2 \alpha_{J}\right) .
\end{aligned}
$$

For $|x / 4|<1$, we find that $e^{w_{k}}=x / 4 \pm i \sqrt{1-(x / 4)^{2}}$, and the corresponding energy $\varepsilon_{k}= \pm \sqrt{-w_{k}^{2}}$ is real.

Now let us solve for the inequality $|x / 4|<1$ analytically for certain simple cases to identify the $k$ modes with purely real single-particle energy $\varepsilon_{k}$. For $\alpha_{J}=0$, one has 

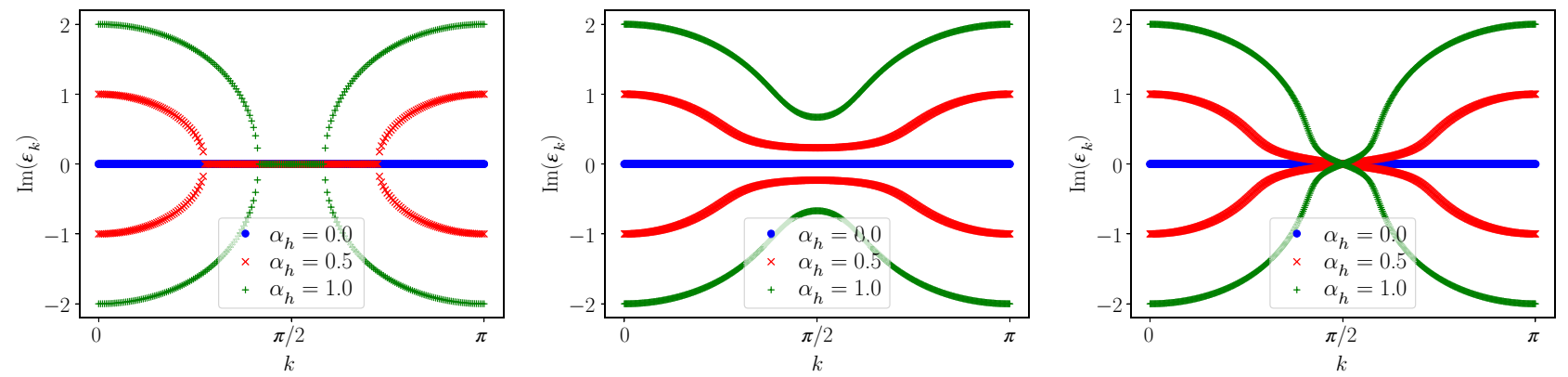

FIG. 11. Imaginary part of the single-particle energy spectrum $\left\{\varepsilon_{k}\right\}$. Left: $J=\pi / 4, h=\pi / 4+i \alpha_{h}$. There exists a finite interval of $k$ modes with real energy [Eq. (B10)] for any noninfinite $\alpha_{h}$. Middle: $J=\pi / 4-0.1, h=\pi / 4+i \alpha_{h}$. Any nonzero $\alpha_{h}$ results in the absence of $k$ modes with purely real energy. Right: $J=\pi / 4, h=\pi / 4-0.1+i \alpha_{h}$. Only the $k$ mode with $k=\pi / 2$ supports purely real energy at any nonzero $\alpha_{h}$.

$|\cos k|<1 / \cosh \left(2 \alpha_{h}\right)$, and for any finite (i.e., noninfinite) $\alpha_{h}$, there is a finite interval of $k$ with purely real energy (see also the left plot of Fig. 11):

$$
\begin{aligned}
k \in I_{k}= & \left(\frac{\pi}{2}-\sin ^{-1}\left[\frac{1}{\cosh \left(2 \alpha_{h}\right)}\right], \frac{\pi}{2}\right. \\
& \left.+\sin ^{-1}\left[\frac{1}{\cosh \left(2 \alpha_{h}\right)}\right]\right) .
\end{aligned}
$$

Within the quasiparticle picture [89], since only those quasiparticle pairs with purely real energy have an infinite lifetime, Eq. (B10) implies the existence of finite density of such quasiparticle pairs, resulting in the volume-law entanglement in long-time-evolved states at any noninfinite $\alpha_{h}$. In particular, the volume-law coefficient of entanglement entropy follows $S_{A} / L_{A} \sim \int_{k \in I_{k}} d k s(k)$, where $I_{k}$ [defined in Eq. (B10)] specifies the interval of $k$ modes with purely real energy; $s(k)$ is the entanglement contributed from the quasiparticle pair with momentum $k$, and is a nonuniversal function determined from the initial state. For large $\alpha_{h}$, since the length of interval $I_{k}$ decays exponentially as $e^{-2 \alpha_{h}}$, the volume-law coefficient $S_{A} / L_{A}$ decays exponentially as well:

$$
\frac{S_{A}}{L_{A}} \sim e^{-b \alpha_{h}}
$$

with $b>0$ a nonuniversal number that depends on the initial state.

Another simple case is $\alpha=\alpha_{J}=\alpha_{h}$, where the corresponding $k$ modes with real energy satisfy $0<k<k_{1}=$ $\cos ^{-1}\{[\cosh (4 \alpha)-3] /[\cosh (4 \alpha)+1]\}$. For large $\alpha$, one finds that $k_{1} \sim e^{-2 \alpha}$, implying the volume-law coefficient

$$
\frac{S_{A}}{L_{A}} \sim e^{-c \alpha},
$$

where $c>0$ is a nonuniversal number that depends on the initial state.

Although here we only discuss two cases (varying $\alpha_{h}$ at fixed $\alpha_{J}=0$ and varying $\alpha=\alpha_{h}=\alpha_{J}$ ), we checked that the condition $\operatorname{Re}(J)=\operatorname{Re}(h)=\pi / 4$ always gives an extensive number of $k$ modes with purely real energy, indicating volume-law entanglement. In strong contrast, any deviation from $\operatorname{Re}(J)=\operatorname{Re}(h)=\pi / 4$ gives an $O(1)$ number of $k$ modes with purely real energy, resulting in the
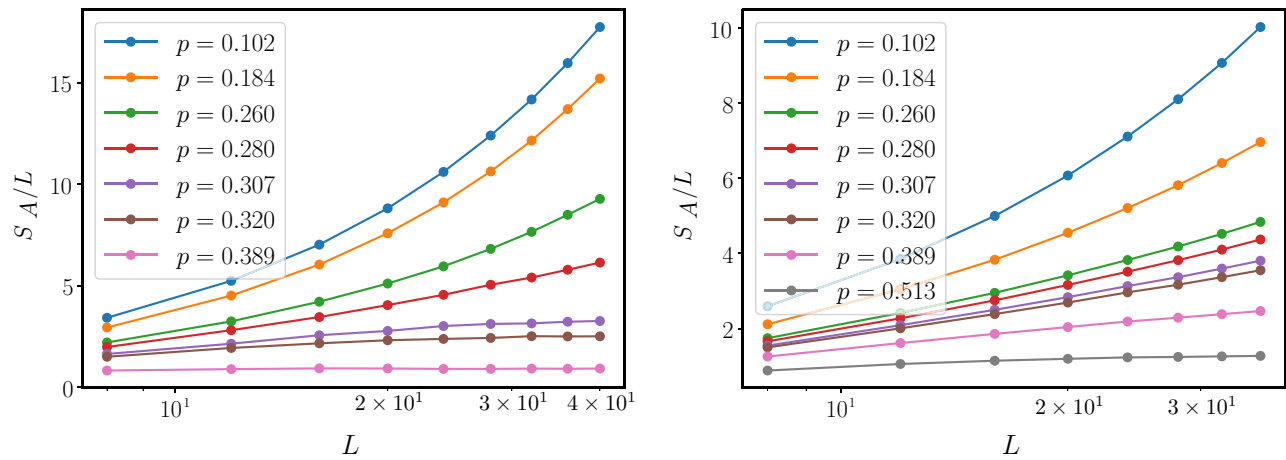

FIG. 12. Left: long-time entanglement entropy $S_{A}$ of a subregion of size $L / 2 \times L$ averaged over $O\left(10^{3}\right)$ random realizations of the unitary circuit defined in Eq. (7). Right: long-time entanglement entropy of a subregion of size $L / 2 \times L$ averaged over $O\left(10^{4}\right)$ random realizations of the rotated nonunitary circuit [Eq. (8)]. The critical point is at $p_{c} \approx 0.28$ for both circuits. 

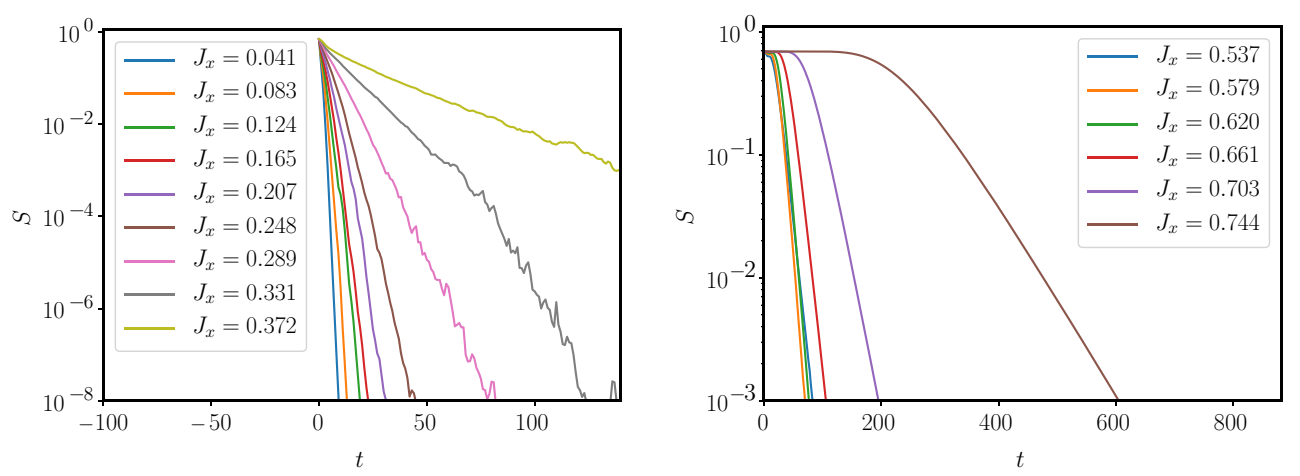

FIG. 13. The entanglement entropy of an ancilla qubit that is initially prepared in the maximally entangled state with a system of size $L=14$, and then evolved with the nonunitary circuit in Eq. (10). Averaging is done over 2000 realizations of the disorder.

absence of volume-law entanglement (see the middle and right plots of Fig. 11).

\section{APPENDIX C: ADDITIONAL DATA FOR THE 2D CLIFFORD CIRCUIT}

Here we present additional data (Fig. 12) on the scaling of entanglement entropy for the late-time states evolved by the unitary circuit [Eq. (7)] and the nonunitary circuit [Eq. (8)]. At the critical point $p_{c} \approx 0.28$, the data are indicative of the scaling $S \sim L \log L$.

\section{APPENDIX D: PURIFICATION DYNAMICS FOR THE ROTATED MBL CIRCUIT DEFINED IN EQ. (10)}

Here we present additional data for the entanglement dynamics of an ancilla qubit that is initially maximally entangled with the system, and then evolved with the nonunitary circuit [Eq. (10)]. For $J_{x} \lesssim 0.4$ (left plot of Fig. 13), the entanglement $S$ of the ancilla qubit decays exponentially with time from its initial value, while for $J_{x} \gtrsim 0.4$ (right plot of Fig. 13), $S$ remains at its initial value for a time that is superlinear in $L$ [see the inset of Fig. 5(d) for scaling with $L]$, followed by an exponential decay.

[1] J. M. Deutsch, Quantum statistical mechanics in a closed system, Phys. Rev. A 43, 2046 (1991).

[2] Mark Srednicki, Chaos and quantum thermalization, Phys. Rev. E 50, 888 (1994).

[3] Mark Srednicki, The approach to thermal equilibrium in quantized chaotic systems, J. Phys. A: Math. Gen. 32, 1163 (1999).

[4] Marcos Rigol, Vanja Dunjko, and Maxim Olshanii, Thermalization and its mechanism for generic isolated quantum systems, Nature 452, 854 (2008).

[5] Luca D'Alessio, Yariv Kafri, Anatoli Polkovnikov, and Marcos Rigol, From quantum chaos and eigenstate thermalization to statistical mechanics and thermodynamics, Adv. Phys. 65, 239 (2016).

[6] D. M. Basko, I. L. Aleiner, and B. L. Altshuler, Metalinsulator transition in a weakly interacting many-electron system with localized single-particle states, Ann. Phys. 321, 1126 (2006).

[7] Vadim Oganesyan and David A. Huse, Localization of interacting fermions at high temperature, Phys. Rev. B 75, 155111 (2007).

[8] Arijeet Pal and David A. Huse, Many-body localization phase transition, Phys. Rev. B 82, 174411 (2010).

[9] David A. Huse, Rahul Nandkishore, and Vadim Oganesyan, Phenomenology of fully many-body-localized systems, Phys. Rev. B 90, 174202 (2014).

[10] Maksym Serbyn, Z. Papić, and Dmitry A. Abanin, Local Conservation Laws and the Structure of the ManyBody Localized States, Phys. Rev. Lett. 111, 127201 (2013).

[11] Rahul Nandkishore and David A. Huse, Many-body localization and thermalization in quantum statistical mechanics, Annu. Rev. Condens. Matter Phys. 6, 15 (2015).

[12] V. Ros, M. Muller, and A. Scardicchio, Integrals of motion in the many-body localized phase, Nucl. Phys. B 891, 420 (2015).

[13] Ehud Altman and Ronen Vosk, Universal dynamics and renormalization in many-body-localized systems, Annu. Rev. Condens. Matter Phys. 6, 383 (2015).

[14] John Z. Imbrie, On many-body localization for quantum spin chains, J. Stat. Phys. 163, 998 (2016).

[15] Fabien Alet and Nicolas Laflorencie, Many-body localization: An introduction and selected topics, C. R. Phys. 19, 498 (2018), Quantum simulation / Simulation quantique.

[16] Dmitry A. Abanin, Ehud Altman, Immanuel Bloch, and Maksym Serbyn, Colloquium: Many-body localization, thermalization, and entanglement, Rev. Mod. Phys. 91, 021001 (2019).

[17] Xiangyu Cao, Antoine Tilloy, and Andrea De Luca, Entanglement in a fermion chain under continuous monitoring, SciPost Phys. 7, 24 (2019).

[18] Brian Skinner, Jonathan Ruhman, and Adam Nahum, Measurement-Induced Phase Transitions in the Dynamics of Entanglement, Phys. Rev. X 9, 031009 (2019).

[19] Yaodong Li Xiao Chen and Matthew P. A. Fisher, Quantum zeno effect and the many-body entanglement transition, Phys. Rev. B 98, 205136 (2018).

[20] Amos Chan, Rahul M. Nandkishore, Michael Pretko, and Graeme Smith, Unitary-projective entanglement dynamics, Phys. Rev. B 99, 224307 (2019).

[21] Michael J. Gullans and David A. Huse, Dynamical Purification Phase Transition Induced by Quantum Measurements, Phys. Rev. X 10, 041020 (2020). 
[22] Michael J. Gullans and David A. Huse, Scalable Probes of Measurement-Induced Criticality, Phys. Rev. Lett. 125, 070606 (2020).

[23] Aidan Zabalo, Michael J. Gullans, Justin H. Wilson, Sarang Gopalakrishnan, David A. Huse, and J. H. Pixley, Critical properties of the measurement-induced transition in random quantum circuits, Phys. Rev. B 101, 060301 (2020).

[24] Soonwon Choi, Yimu Bao, Xiao-Liang Qi, and Ehud Altman, Quantum Error Correction in Scrambling Dynamics and Measurement-Induced Phase Transition, Phys. Rev. Lett. 125, 030505 (2020).

[25] Qicheng Tang and W. Zhu, Measurement-induced phase transition: A case study in the nonintegrable model by density-matrix renormalization group calculations, Phys. Rev. Res. 2, 013022 (2020).

[26] Yaodong Li Xiao Chen and Matthew P. A. Fisher, Measurement-driven entanglement transition in hybrid quantum circuits, Phys. Rev. B 100, 134306 (2019).

[27] M. Szyniszewski, A. Romito, and H. Schomerus, Entanglement transition from variable-strength weak measurements, Phys. Rev. B 100, 064204 (2019).

[28] Lei Zhang, Justin A. Reyes, Stefanos Kourtis, Claudio Chamon, Eduardo R. Mucciolo, and Andrei E. Ruckenstein, Nonuniversal entanglement level statistics in projection-driven quantum circuits, Phys. Rev. B 101, 235104 (2020).

[29] Shimpei Goto and Ippei Danshita, Measurement-induced transitions of the entanglement scaling law in ultracold gases with controllable dissipation, Phys. Rev. A 102, 033316 (2020).

[30] Chao-Ming Jian, Yi-Zhuang You, Romain Vasseur, and Andreas W. W. Ludwig, Measurement-induced criticality in random quantum circuits, Phys. Rev. B 101, 104302 (2020).

[31] Yimu Bao, Soonwon Choi, and Ehud Altman, Theory of the phase transition in random unitary circuits with measurements, Phys. Rev. B 101, 104301 (2020).

[32] Yaodong Li, Xiao Chen, Andreas W. W. Ludwig, and Matthew Fisher, Conformal invariance and quantum nonlocality in hybrid quantum circuits, arXiv:2003.12721, (2020).

[33] Nicolai Lang and Hans Peter Büchler, Entanglement transition in the projective transverse field ising model, Phys. Rev. B 102, 094204 (2020).

[34] Adam Nahum and Brian Skinner, Entanglement and dynamics of diffusion-annihilation processes with majorana defects, Phys. Rev. Res. 2, 023288 (2020).

[35] Shengqi Sang and Timothy H. Hsieh, Measurement protected quantum phases, arXiv:2004.09509 (2020).

[36] Ali Lavasani, Yahya Alavirad, and Maissam Barkeshli, Measurement-induced topological entanglement transitions in symmetric random quantum circuits, Nat. Phys. 17, 342 (2021).

[37] Ali Lavasani, Yahya Alavirad, and Maissam Barkeshli, Topological order and criticality in $(2+1) \mathrm{d}$ monitored random quantum circuits, arXiv:2011.06595 (2020).

[38] Matteo Ippoliti, Michael J. Gullans, Sarang Gopalakrishnan, David A. Huse, and Vedika Khemani, Entanglement
Phase Transitions in Measurement-Only Dynamics, Phys. Rev. X 11, 011030 (2021).

[39] Carlos M. Duque, Hong-Ye Hu, Yi-Zhuang You, Ruben Verresen, and Romain Vasseur, Topological and symmetry-enriched random quantum critical points, arXiv:2008.02285 (2020).

[40] Xhek Turkeshi, Rosario Fazio, and Marcello Dalmonte, Measurement-induced criticality in $(2+1)$-dimensional hybrid quantum circuits, Phys. Rev. B 102, 014315 (2020).

[41] Yohei Fuji and Yuto Ashida, Measurement-induced quantum criticality under continuous monitoring, Phys. Rev. B 102, 054302 (2020).

[42] Oliver Lunt and Arijeet Pal, Measurement-induced entanglement transitions in many-body localized systems, Phys. Rev. Res. 2, 043072 (2020).

[43] M. Szyniszewski, A. Romito, and H. Schomerus, Universality of Entanglement Transitions from Stroboscopic to Continuous Measurements, Phys. Rev. Lett. 125, 210602 (2020).

[44] Sagar Vijay, Measurement-driven phase transition within a volume-law entangled phase, arXiv:2005.03052 (2020).

[45] Javier Lopez-Piqueres, Brayden Ware, and Romain Vasseur, Mean-field entanglement transitions in random tree tensor networks, Phys. Rev. B 102, 064202 (2020).

[46] Lukasz Fidkowski, Jeongwan Haah, and Matthew B. Hastings, How dynamical quantum memories forget, Quantum 5, 382 (2021).

[47] Adam Nahum, Sthitadhi Roy, Brian Skinner, and Jonathan Ruhman, Measurement and entanglement phase transitions in all-to-all quantum circuits, on quantum trees, and in landau-ginsburg theory, arXiv:2009.11311 (2020).

[48] Yimu Bao, Soonwon Choi, and Ehud Altman, Symmetry enriched phases of quantum circuits, arXiv:2102.09164 (2021).

[49] Baidyanath Misra and E. C. George Sudarshan, The zeno's paradox in quantum theory, J. Math. Phys. 18, 756 (1977).

[50] Xiao Chen, Yaodong Li, Matthew P. A. Fisher, and Andrew Lucas, Emergent conformal symmetry in nonunitary random dynamics of free fermions, Phys. Rev. Res. 2, 033017 (2020).

[51] Qicheng Tang, Xiao Chen, and W. Zhu, Quantum criticality in non-unitary dynamics of $2+1 \mathrm{~d}$ free fermions, arXiv:2101.04320 (2021).

[52] Chao-Ming Jian, Bela Bauer, Anna Keselman, and Andreas W. W. Ludwig, Criticality and entanglement in non-unitary quantum circuits and tensor networks of non-interacting fermions, arXiv:2012.04666 (2020).

[53] Chunxiao Liu, Pengfei Zhang, and Xiao Chen, Nonunitary dynamics of sachdev-ye-kitaev chain, SciPost Phys. 10, 48 (2021).

[54] Shao-Kai Jian, Zhi-Cheng Yang, Zhen Bi, and Xiao Chen, Yang-lee edge singularity triggered entanglement transition, arXiv:2101.04115 (2021).

[55] M Akila, D Waltner, B Gutkin, and T Guhr, Particle-time duality in the kicked ising spin chain, J. Phys. A: Math. Theor. 49, 375101 (2016). 
[56] Bruno Bertini, Pavel Kos, and Tomaž Prosen, Exact Spectral Form Factor in a Minimal Model of ManyBody Quantum Chaos, Phys. Rev. Lett. 121, 264101 (2018).

[57] Bruno Bertini, Pavel Kos, and Tomaž Prosen, Entanglement Spreading in a Minimal Model of Maximal Many-Body Quantum Chaos, Phys. Rev. X 9, 021033 (2019).

[58] Amos Chan, Andrea De Luca, and J. T. Chalker, Spectral Statistics in Spatially Extended Chaotic Quantum ManyBody Systems, Phys. Rev. Lett. 121, 060601 (2018).

[59] Amos Chan, Andrea De Luca, and J. T. Chalker, Spectral lyapunov exponents in chaotic and localized many-body quantum systems, arXiv:2012.05295 (2020).

[60] Lorenzo Piroli, Bruno Bertini, J. Ignacio Cirac, and Tomaž Prosen, Exact dynamics in dual-unitary quantum circuits, Phys. Rev. B 101, 094304 (2020).

[61] Pavel Kos, Bruno Bertini, and Tomaž Prosen, Correlations in Perturbed Dual-Unitary Circuits: Efficient Path-Integral Formula, Phys. Rev. X 11, 011022 (2021).

[62] John Napp, Rolando L. La Placa, Alexander M. Dalzell, Fernando GSL Brandao, and Aram W. Harrow, Efficient classical simulation of random shallow $2 \mathrm{~d}$ quantum circuits, arXiv:2001.00021 (2019).

[63] Matteo Ippoliti and Vedika Khemani, Postselection-Free Entanglement Dynamics via Spacetime Duality, Phys. Rev. Lett. 126, 060501 (2021).

[64] Pedro Ponte, Anushya Chandran, Z. Papić, and Dmitry A. Abanin, Periodically driven ergodic and manybody localized quantum systems, Ann. Phys. 353, 196 (2015).

[65] Dmitry A. Abanin, Wojciech De Roeck, and François Huveneers, Theory of many-body localization in periodically driven systems, Ann. Phys. 372, 1 (2016).

[66] Liangsheng Zhang, Vedika Khemani, and David A. Huse, A floquet model for the many-body localization transition, Phys. Rev. B 94, 224202 (2016).

[67] Adam Nahum, Jonathan Ruhman, Sagar Vijay, and Jeongwan Haah, Quantum Entanglement Growth under Random Unitary Dynamics, Phys. Rev. X 7, 031016 (2017).

[68] Vedika Khemani, Ashvin Vishwanath, and David A. Huse, Operator Spreading and the Emergence of Dissipative Hydrodynamics under Unitary Evolution with Conservation Laws, Phys. Rev. X 8, 031057 (2018).

[69] Adam Nahum, Sagar Vijay, and Jeongwan Haah, Operator Spreading in Random Unitary Circuits, Phys. Rev. X 8, 021014 (2018).

[70] Tibor Rakovszky, Frank Pollmann, and C. W. von Keyserlingk, Diffusive Hydrodynamics of Out-Of-Time-Ordered Correlators with Charge Conservation, Phys. Rev. X 8, 031058 (2018).

[71] C. W. von Keyserlingk, Tibor Rakovszky, Frank Pollmann, and S. L. Sondhi, Operator Hydrodynamics, OTOCs, and Entanglement Growth in Systems Without Conservation Laws, Phys. Rev. X 8, 021013 (2018).

[72] Tianci Zhou and Xiao Chen, Operator dynamics in a brownian quantum circuit, Phys. Rev. E 99, 052212 (2019).

[73] Xiao Chen and Tianci Zhou, Quantum chaos dynamics in long-range power law interaction systems, Phys. Rev. B 100, 064305 (2019).
[74] Tianci Zhou and Adam Nahum, Emergent statistical mechanics of entanglement in random unitary circuits, Phys. Rev. B 99, 174205 (2019).

[75] Hiroshi Betsuyaku, Study of One-Dimensional XY Model by the Transfer-Matrix Method, Phys. Rev. Lett. 53, 629 (1984).

[76] Thomas Guhr, Axel Müller-Groeling, and Hans A. Weidenmüller, Random-matrix theories in quantum physics: Common concepts, Phys. Rep. 299, 189 (1998).

[77] Jordan S. Cotler, Guy Gur-Ari, Masanori Hanada, Joseph Polchinski, Phil Saad, Stephen H. Shenker, Douglas Stanford, Alexandre Streicher, and Masaki Tezuka, Black holes and random matrices, J. High Energy Phys. 2017, 118 (2017).

[78] Jan Šuntajs, Janez Bonča, Tomaž Prosen, and Lev Vidmar, Quantum chaos challenges many-body localization, Phys. Rev. E 102, 062144 (2020).

[79] Abhishodh Prakash, J. H. Pixley, and Manas Kulkarni, Universal spectral form factor for many-body localization, Phys. Rev. Res. 3, L012019 (2021).

[80] Patrycja Łydżba, Marcos Rigol, and Lev Vidmar, Eigenstate Entanglement Entropy in Random Quadratic Hamiltonians, Phys. Rev. Lett. 125, 180604 (2020).

[81] Philip George Harper, The general motion of conduction electrons in a uniform magnetic field, with application to the diamagnetism of metals, Proc. Phys. Soc. Sect. A 68, 879 (1955).

[82] M. Ya Azbel, Quantum Particle in One-Dimensional Potentials with Incommensurate Periods, Phys. Rev. Lett. 43, 1954 (1979).

[83] Serge Aubry and Gilles André, Analyticity breaking and anderson localization in incommensurate lattices, Ann. Israel Phys. Soc. 3, 18 (1980).

[84] P. W. Anderson, Absence of diffusion in certain random lattices, Phys. Rev. 109, 1492 (1958).

[85] A. Chandran and C. R. Laumann, Localization and Symmetry Breaking in the Quantum Quasiperiodic Ising Glass, Phys. Rev. X 7, 031061 (2017).

[86] Ming-Chiang Chung and Ingo Peschel, Density-matrix spectra of solvable fermionic systems, Phys. Rev. B 64, 064412 (2001).

[87] Siew-Ann Cheong and Christopher L. Henley, Manybody density matrices for free fermions, Phys. Rev. B 69 , 075111 (2004).

[88] Ingo Peschel, Calculation of reduced density matrices from correlation functions, J. Phys. A: Math. Gen. 36, L205 (2003).

[89] Pasquale Calabrese and John Cardy, Evolution of entanglement entropy in one-dimensional systems, J. Stat. Mech.: Theory and Exp. 2005, P04010 (2005).

[90] Daniel Gottesman, Class of quantum error-correcting codes saturating the quantum hamming bound, Phys. Rev. A 54, 1862 (1996).

[91] Daniel Gottesman, The heisenberg representation of quantum computers, arXiv:quant-ph/9807006 (1998).

[92] Scott Aaronson and Daniel Gottesman, Improved simulation of stabilizer circuits, Phys. Rev. A 70, 052328 (2004).

[93] Anushya Chandran and C. R. Laumann, Semiclassical limit for the many-body localization transition, Phys. Rev. B 92, 024301 (2015). 
[94] H. Casini and M. Huerta, Renormalization group running of the entanglement entropy of a circle, Phys. Rev. D 85, 125016 (2012).

[95] Sidney Redner and H Eugene Stanley, Anisotropic bond percolation, J. Phys. A: Math. Gen. 12, 1267 (1979).

[96] Don N. Page, Average Entropy of a Subsystem, Phys. Rev. Lett. 71, 1291 (1993).

[97] Leonid V. Keldysh et al., Diagram technique for nonequilibrium processes, Sov. Phys. JETP 20, 1018 (1965).

[98] L. P. Kadanoff and G. Baym, Quantum Statistical Mechanics: Green's Function Methods in Equilibrium and Nonequilibrium Problems. Frontiers in Physics: Lecture note and reprint series, A. W.A. Benjamin, (1962).

[99] Liang Jiang, Gavin K. Brennen, Alexey V. Gorshkov, Klemens Hammerer, Mohammad Hafezi, Eugene Demler, Mikhail D. Lukin, and Peter Zoller, Anyonic interferometry and protected memories in atomic spin lattices, Nat. Phys. 4, 482 (2008).

[100] Dmitry A. Abanin and Eugene Demler, Measuring Entanglement Entropy of a Generic Many-Body System with a Quantum Switch, Phys. Rev. Lett. 109, 020504 (2012).

[101] M. Müller, I. Lesanovsky, H. Weimer, H. P. Büchler, and P. Zoller, Mesoscopic Rydberg Gate Based on Electromagnetically Induced Transparency, Phys. Rev. Lett. 102, 170502 (2009).

[102] R. Vasseur, S. A. Parameswaran, and J. E. Moore, Quantum revivals and many-body localization, Phys. Rev. B 91, 140202 (2015).
[103] Jiachen Li, Tomaž Prosen, and Amos Chan, Spectral statistics of non-hermitian matrices and dissipative quantum chaos, arXiv:2103.05001 (2021).

[104] J. T. Chalker and P. D. Coddington, Percolation, quantum tunnelling and the integer hall effect, J. Phys. C: Solid State Phys. 21, 2665 (1988).

[105] Vedika Khemani, Matteo Ippoliti, and Tibor Rakovszky, Fractal, logarithmic and volume-law entangled nonthermal steady states via spacetime duality, arXiv:2103. 06873 (2021). (appeared on arXiv concurrently with the version-1 of our preprint arXiv:2103.06356v1).

[106] The first version of this preprint on arXiv mentioned that a nonunitary circuit discussed in the Section VIB of Ref. [26] shows an entanglement transition while possessing a spacetime dual unitary that is always ergodic. However, this statement is incorrect. The true statement is that, when the dual of the said nonunitary circuit is unitary, the nonunitary circuit in fact does not host an entanglement transition either (this phenomena is similar to the absence of transition in the circuit briefly mentioned in Sec. IV, which is related to an infinitely anisotropic bond percolation problem, i.e., where bonds only along one direction on the spacetime lattice are being removed). We thank Matteo Ippoliti, Tibor Rakovszky, and Vedika Khemani for pointing this out to us.

[107] John Towns, Timothy Cockerill, Maytal Dahan, Ian Foster, Kelly Gaither, Andrew Grimshaw, Victor Hazlewood, Scott Lathrop, Dave Lifka, Gregory D. Peterson, Ralph Roskies, J. Ray Scott, and Nancy Wilkins-Diehr, Xsede: Accelerating scientific discovery, Comput. Sci. Eng. 16, 62 (2014). 